\title{
ZnO Quantum Dots-Graphene composites: Formation Mechanism and Enhanced Photocatalytic Activity for Degradation of Methyl Orange Dye
}

\author{
Ahmad Tayyebi $^{1}$, Mohammad outokesh $^{1 *}$, Meysam Tayebi $^{2}$, Azizollah Shafikhani $^{3}$, S. Sevinç Şengör ${ }^{4}$
}

\author{
${ }^{1}$ Department of Energy Engineering, Sharif University of Technology, Azadi Ave. P.O. Box: 113658639, Tehran, Iran. \\ ${ }^{2}$ Chemical Engineering Department, Faculty of Engineering, Ferdowsi University of Mashhad \\ ${ }^{3}$ Departmentof Physics, AlZahra University, Tehran, 1993893973, Iran. \\ ${ }^{4}$ Southern Methodist University, Department of Civil and Environmental Engineering, Dallas, TX 75275, USA \\ *Corresponding author's email address: Outokesh@sharif.edu
}

(C) 2015. This manuscript version is made available under the Elsevier user license http://www.elsevier.com/open-access/userlicense/1.0/ 
Abstract: The current study demonstrates homogenous decorating of zinc oxide quantum dots (QDs) onto graphene oxide (GO) surface via simple chemical method. The AFM image exhibited that the prepared graphene was $0.8 \mathrm{~nm}$ thick and hence practically monolayer. Average size of the $\mathrm{ZnO}$ QDs was estimated by transmission electron microscopy around $3 \mathrm{~nm}$. Instrumental and chemical analyses demonstrated formation of a strong bond between $\mathrm{ZnO}$ QDs and GO, through CO-Zn and C-Zn bridges. The UV-visible spectra displayed that the introduction of graphene sheets to $\mathrm{ZnO}$ QDs resulted in higher absorption intensity of UV as well as widening of adsorption window toward visible light for $\mathrm{ZnO}-$ Graphene due to chemical bond between $\mathrm{ZnO}$ QDS and graphene surface. Results showed that adding of graphene up to $30 \%$ can improve resistance of $\mathrm{ZnO}$ against acids however for keeping the activity of catalyst, the recommended $\mathrm{pH}$ is near neutral ( $\mathrm{pH} \approx 6-7.2$ ). In addition, the presence of graphene on the surface of the $\mathrm{ZnO}$ could significantly suppress the photocorrosion effect. The $\mathrm{ZnO}-$ Graphene hybrids indicated enhanced photocatalytic activity for degradation of methyl orange (MO) with the following order: $\mathrm{ZnO}-5 \%$ Graphene > $\mathrm{ZnO}-10 \%$ Graphene > $\mathrm{ZnO}$ QDs > $\mathrm{ZnO} 30 \%$-Graphene. This enhancement of photocatalytic activity may be attributed to the extended absorption of visible light, reducing of electron-hole recombination rate, and adsorption of MO molecules onto the huge surface area of graphene, where they are kept at vicinity of $\mathrm{ZnO}$ for decomposition.

Keywords: ZnO-Graphene; Enhanced photocatalysis; Formation mechanism; Visible photocatalytic activity, Photocorrosion 
Introduction. Recent global warming that has decreased the amounts of atmospheric precipitations in some geographical areas, accompanied with the rapid growth of the world population, has raised concern about depleting of the clean water resources[1]. Thus, treatment, and reuse of wastewater has become an important global issue. Between the three types of wastewater, namely industrial, agricultural and municipal, the first one is the easiest to treat, because in any kind of industry, number of the chemical agents that pollute the water is limited. Nowadays, in industrialized countries, post processing of the waste water is commonplace, because the rate of production of the effluents is gigantic, and the environmental concern adds to the significance of the problem[2].

As for treatment of the industrial effluents, so far, a great deal of technologies has been devised[3]. One of the finest techniques for decontamination of the wastewater, which recently has attracted much interests is photo-degradation of the toxic pollutants by nanocatalysts[4]. Up to now, several metal oxide semiconductors have been tested as photocatalyst for this purpose, including: $\mathrm{TiO}_{2}, \mathrm{SnO}_{2}, \mathrm{WO}_{3}, \mathrm{Fe}_{3} \mathrm{O}_{4}$, and eventually $\mathrm{ZnO}$ [5-7]. Among these materials, zinc oxide presents numerous advantages such as: availability, non-toxicity, cost efficiency, and thermal stability [8]. As regard to only being active in the UV region of the solar light, and thus absorbing only $5 \%$ of the sunlight energy, extending of the band gap of $\mathrm{ZnO}$ into the visible region, and hence improving of its photocatalytic efficiency is of great importance[9, 10]. One of the suggested methods for this purpose is decorating of $\mathrm{ZnO}$ nanoparticles (NPs) on a substrate with high surface area such as zeolite, nanoclay or carbon-based materials [11-13]. The aforementioned carbon-based materials are divided into two categories:1-Conventional, including graphite, carbon black, and activated carbon which have been studied extensively, and 2- New family comprising carbon nanotube (CNT), fullerenes, and most recently graphene. Since their emerges in the past few years, the new family have presented themselves as attractive hybridizing agents for synthesis of photocatalysts owing to their exceptional electronic properties, and the adsorption capacity[14-16]. Graphene starred for the first time in the scientific sky in 2004[17] and due to its unique electronic, optoelectronic, and physical properties, opened new windows in synthesis of advanced 
materials. Graphene-based hybrids have been widely employed in various applications including polymer-composite[18], drug delivery[19], sensor fabrication [20], catalysis[21], and nanoelectronic[22].

Currently, most of the graphene derivatives (G-Ds) are prepared from graphene oxide (GO) as the starting material. This is due to the fact that $\mathrm{GO}$ is an intermediate material in the synthesis of graphene, and its usage can shorten synthesis time of the G-Ds considerably[23]. Graphene oxide is a lamellar material with epoxy $(\mathrm{C}-\mathrm{O}-\mathrm{C})$, hydroxyl $(\mathrm{OH})$, and carboxyl $(\mathrm{COOH})$ groups, that its groups can act as nucleation sites for growth of semiconductor nanoparticles[24]. Recently, graphene-based semiconductor photocatalysts have received a great deal of attention owing to their high catalytic performance[25, 26]. Moreover, due to the existence of oxygen functionalities on its surface, and the enormous surface area, deposition of semiconductors NPs on the GO is homogeneous[27]. Furthermore, Deposition of the nanoparticles on the GO also prevents restacking of the graphene sheets during post-reduction of the GO[28].

So far, several researchers investigated synthesis of $\mathrm{ZnO}-$ Graphene hybrid for different applications. Nearly in all of those works, ZnO-Graphene showed an enhanced activity compared to the pristine $\mathrm{ZnO}[8,14,29]$. Bai and coworkers synthesized $\mathrm{ZnO}-$ Graphene using in-situ reduction of graphene oxide and utilized it for photo degradation of methylene blue. The hybrid showed 4.6 times higher efficiency under visible light compared to pure $\mathrm{ZnO}[14]$. Lv et al. prepared $\mathrm{ZnO}-$ Graphene-CNT triple composite to improve the low electric conductivity of the ZnO-Graphene hybrid. Under UV irradiation, the new composite showed a maximum degradation efficiency equal to $96 \%$, which was higher than that of the $\mathrm{ZnO}-$ Graphene hybrid (88\%)[30]. Because of the efficient electron-hole separation, notable electron conductivity, and high surface area of graphene as substrate, incorporation of graphene and semiconductors in a composite is turned out to lead to conspicuous photocatalytic activity.

Semiconductor quantum dots (QDs) present size-dependent band gap shift[31] resulting in interesting optical properties due to the quantum confinement effect. The smaller ZnO NPs are, and 
the narrower size distribution they have, the higher surface for catalytic reaction they achieve, and the wider band gap and photocatalytic performance they attain[32]. Zubir et al. and Song et al. proposed that $\mathrm{ZnO}$ QDs are decorated on the surface of GO via covalent connection between $\mathrm{Zn}^{2+}(\mathrm{ZnO})$ or $\mathrm{Zn}^{2+}$ and oxygen functionalities on the surface of GO[33]. Hsiao et al. showed that the functional groups can be efficiently grafted onto surface of graphene sheets using epoxy ringopening reaction[34]. Wang et al. demonstrated that metal oxide may not be grafted onto pristine graphene unless surface functionalization was provided beforehand [35]. Ma et al. found that dangling bonds and defects on the surface of graphene could act as a bridge between $\mathrm{ZnO}$ and the graphene sheet[36]. Despite great deal of attempts that have been made for clarification of mechanism of anchoring of $\mathrm{ZnO}$ onto graphene surface, there is still a paucity of reliable information about this linkage.

The current study is aimed at synthesis of the $\mathrm{ZnO}-$ Graphene composite with the $\mathrm{ZnO}$ particles in the range of few nanometers, elucidation of their formation mechanism, and application of the obtained composite for photocatalytic degradation of methyl orange. The study particularly intends to investigate widening of the band gap of $\mathrm{ZnO}$ QDs from UV to visible region, when it is compounded with graphene.

\section{Experimental Methods}

2.1. Materials. Natural flake graphite, sulfuric acid (98\%), hydrochloric acid (37\%), hydrogen peroxide (30\%), sodium nitrate, anhydrous dimethyl formamide (DMF), zinc acetate and potassium permanganate were purchased from Merck, AG, Germany and used without purification.

2.1. Preparation of graphene oxide. Graphene Oxide was prepared from highly pure graphite using the modified Hummers method [37]. In a typical procedure, a mixture of $2 \mathrm{~g}$ graphite powder and $2 \mathrm{~g}$ sodium nitrate was dispersed in $45 \mathrm{~cm}^{3}$ of $95 \%$ (w/w) sulfuric acid. While the temperature was maintained below $5{ }^{0} \mathrm{C}$ using ice bath, $6 \mathrm{~g}$ potassium permanganate was gradually added to the 
dispersion within $2 \mathrm{~h}$. Then the mixture was stirred for $30 \mathrm{~min}$, and $100 \mathrm{~mL}$ distillated water was slowly added to it. At this stage, temperature was increased to $95{ }^{0} \mathrm{C}$, and the mixture was maintained at that temperature for another $30 \mathrm{~min}$. Afterward, the hot mixture was cooled down to $40{ }^{\circ} \mathrm{C}$ by adding $300 \mathrm{~mL}$ distillated water, and reaction was terminated by using $20 \mathrm{~mL}$ of a $30 \%$ (w/v) hydrogen peroxide solution. The obtained mixture was filtered, washed with diluted $\mathrm{HCl}$ to remove metal ions, and repeatedly washed (with distilled water) and centrifuged until the $\mathrm{pH}$ become 5. Finally prepared GO was dried at ambient temperature.

2.2. Synthesis of $\mathrm{ZnO}$-Graphene composite. $\mathrm{ZnO}$-Graphene hybrid with different amount of graphene was synthesized by slight modification of Son et al. method[33]. The procedure was started by uniform dispersion of calculated amount of GO in $100 \mathrm{~mL}$ DMF by the aid of ultrasonication (Elmasonic, $\mathrm{S} 30 \mathrm{H}$, country) for $15 \mathrm{~min}$ to prepare 5, 10, and $30 \%$ of graphene in ZnO-Graphene composites. Simultaneously, $1 \mathrm{~g}$ of zinc acetate was dissolved in a mixture of 100 $\mathrm{mL}$ DMF and $20 \mathrm{~mL}$ deionized water. The GO solution then was gradually mixed with zinc acetate solution by stirring. The obtained mixture was then heated to $95{ }^{0} \mathrm{C}$ and maintained at that temperature for $5 \mathrm{~h}$. During this period, the color of the formed $\mathrm{ZnO}-\mathrm{Graphene}$ hybrid was altered to white-greyish. The mixed solution was then repeatedly washed with ethanol, and finally with water. The $\mathrm{ZnO}-$ Graphene composite was obtained by drying of the final centrifuged product at 55 ${ }^{0} \mathrm{C}$.

2.3. Characterization. Morphological images of the $\mathrm{GO}$ nanosheets and $\mathrm{ZnO}$-Graphene composite were obtained by means of low and high-resolution transmission electron microscopy (TEM / HRTEM, JEOL, JEM-2100, Japan). The employed HRTEM also supported the selected area electron diffraction (SAED) analysis. Evidence for complete exfoliation of the graphene (i.e. formation of single layer product) was provided by atomic force microscopy (AFM, Park Scientific CP-Research model, VEECO) which worked in the tapping mode, with frequency of $320 \mathrm{kHz}$, and 
by using a $20 \mathrm{~nm}$ thick silicone tip. Samples for AFM imaging were prepared by drop casting of a dilute GO suspension $(0.01 \mathrm{mg} / \mathrm{mL})$ onto a cleaved mica substrate.

The current study exploited five methods for identifying of the chemical and crystallographic structure of the prepared materials. The first method was Raman spectroscopy (SENTERRA, BRUKER, Germany) that was performed at room temperature by a $532 \mathrm{~nm} \mathrm{Nd-}$ YAG excitation laser. The second technique was energy dispersive X-ray analysis (EDX, JEOL, JEM-2100, and Japan) which provided the elemental analysis of the samples. The X-ray photoelectron spectroscopy (XPS) as the third technique revealed the chemical states of the GO before and after its reduction to graphene. The XPS instrument was equipped with a hemispherical analyzer for data acquisition, and an $\mathrm{Al} \mathrm{K}_{\alpha} \mathrm{X}$-ray source operated at pressure below $10^{-7} \mathrm{~Pa}$. The XPS peaks were fitted by Gaussian components model after Shirley background subtraction. Two other employed analysis techniques included X- ray diffractometry (Rigaku Miniflex XRD, Texas, USA), and Fourier transforms infrared spectroscopy (FTIR, Perkin-Elmer, Spectrum RX, USA).

Optical characteristics of the GO suspension $\left(0.01 \mathrm{mg} / \mathrm{cm}^{3}\right)$ were studied by a UV-Visible spectrophotometer (Perkin Elmer UV-Vis-NIR model Lambda 950) in the wavelength range of 200-700 nm.

In order to study the thermal stability and composition of prepared materials, thermogravimetric analysis (TGA) was performed under air atmosphere (TGA/DSC 1, METTLER TOLEDO, Switzerland). A heating rate of $10^{\circ} \mathrm{C} / \mathrm{min}$ was utilized.

2.4. Chemical Stability and Sedimentation Measurements: Dissolution of ZnO QDs and ZnOGraphene with different amount of graphene was investigated in a glass reactor at room temperature and different $\mathrm{pH}$ values including acidic $(\mathrm{pH} 1.0)$, mildly acidic $(\mathrm{pH} 3.0)$, neutral $(\mathrm{pH}$ 6.0-7.2), and neutral media ( $\mathrm{pH} 7.1)$. For this reason, same amount of samples $(0.1 \mathrm{gr} / \mathrm{L})$ of $\mathrm{ZnO}$ QDs, ZnO-5\% Graphene, ZnO-10 \% Graphene, and $\mathrm{ZnO}-30 \%$ Graphene were dispersed in water solution and after 15 min ultrasonication at different time, sample were collected from supernatant. 
The solid component was removed by $0.2 \mu \mathrm{m}$ membrane filter and centrifugation process. The concentration of Zinc $\left(\mathrm{Zn}^{2+}\right)$ ions was measured by a Varian atomic adsorption spectrometer (AAS).

The stability of obtained materials at $\mathrm{pH} 7$ was determined by monitoring sedimentation by a Perkin-Elmer Lambada20 UV-vis spectrometer. The change in the optical absorbance at $375 \mathrm{~nm}$ was obtained as a function of time.

2.5. Kinetics of the MO Adsorption. Batch-mode adsorption kinetics of MO by ZnO QDs and ZnO-Graphene were studied by adding $10 \mathrm{mg}$ of each adsorbent to $10 \mathrm{~mL}$ of a $20 \mathrm{ppm}$ MO solution that was placed in a conical flask. Samples of the solution were withdrawn at different time interval, and analysed by UV-visible spectrometer (Perkin Elmer UV-Vis-NIR model Lambda 950). The temporal loading of MO onto adsorbent was obtained from the following relation:

$$
q_{t}=\frac{\left(C_{0}-C_{t}\right) V}{m}
$$

where $\mathrm{C}_{0}(\mathrm{ppm})$ and $\mathrm{C}_{\mathrm{t}}(\mathrm{ppm})$ show concentration of the $\mathrm{MO}$ at time zero and time "t, min ", V $(\mathrm{mL})$ stands for volume of the solution, and $\mathrm{m}(\mathrm{g})$ is the mass of the adsorbent.

2.6. Photocatalytic activity. The photocatalytic activities of $\mathrm{ZnO}$ QDs and $\mathrm{ZnO}-$ Graphene were studied by examining degradation rate of Methyl Orange (MO), in the presence of the above adsorbents under ultraviolet (UV) and visible light irradiation. The photocatalysis experiments were carried out in an open wide glass reactor using a high-pressure $100 \mathrm{~W}$ mercury lamp (B 100 AP, UVP) with the wavelength of $365 \mathrm{~nm}$ as the UV source, and a $175 \mathrm{~W}$ metal halide lamp $(\lambda>420$ $\mathrm{nm})$ as the visible light supplier. Both of the lamps were placed $10 \mathrm{~cm}$ away from the reactor. About $50 \mathrm{mg}$ of $\mathrm{ZnO}$ QDs or $\mathrm{ZnO}$-Graphene was dispersed in $100 \mathrm{~mL}$ of a $1.5 \times 10^{-4} \mathrm{M}$ aqueous 
MO solution $(\mathrm{pH}=6.5)$. Prior the lightening of the lamps, suspensions were magnetically stirred for $30 \mathrm{~min}$ in dark to attain the adsorption-desorption equilibrium. At this moment, concentration of $\mathrm{MO}$ was assigned as $\mathrm{C}_{0}$. After onset of the irradiation, $3 \mathrm{~mL}$ of liquid was withdrawn in each time interval, and analysed by a UV-visible spectrometer (Perkin Elmer UV-Vis-NIR model Lambda 950).

3. Result and Discussion. Figure 1a shows AFM image of the GO on a freshly cleaved mica surface. The height profile of the line marked in the AFM image indicates monolayer graphene sheets with $0.8 \mathrm{~nm}$ thickness. It is widely accepted that due to the presence of oxygen functionalities on the surface of GO, its thickness is around $0.8-1.1 \mathrm{~nm}$, which is somewhat thicker than the $0.4 \mathrm{~nm}$ thickness of graphene sheet[38]. Figure $1 \mathrm{~b}$ displays the TEM image of the GO in which wrinkling and overlapping of the sheets is evident. Transparent areas in this figure exhibit monolayer graphene. Existence of six resolved spots in each depicted ring, is a clear indication of the hexagonal lattice of graphene in SAED pattern of GO in inset Figure 1b.The UV absorption spectrum of GO in Figure 1c reveals a distinct peak at $231 \mathrm{~nm}$ corresponding to $\pi-\pi^{*}$ transitions for aromatic C-C and a shoulder at $302 \mathrm{~nm}$ assigned to $\mathrm{n}-\pi^{*}$ transitions of $\mathrm{C}=\mathrm{O}$ bonds[39].

\section{Figure 1}

3.1. Morphology. Figure $S 1$ in supplementary information shows low magnification TEM image of $\mathrm{ZnO}-\mathrm{Graphene}$ and corresponding EDX analysis in which the materials compositions of both $\mathrm{ZnO}$ and graphene phases are depicted.

Low magnification TEM image of $\mathrm{ZnO}-$ Graphene is displayed in Figure 2a, in which the border of the graphene sheet is marked by the dashed red line. The ZnO QDs are homogenously distributed on the surface of graphene with average size of $3 \mathrm{~nm}$ (From histogram of Figure S2 in supplementary information). The homogeneity of $\mathrm{ZnO}$ distribution can be attributed to the presence 
of enumerable oxygen functionalities on the surface of GO, which provide anchoring sites for immobilization of the $\mathrm{ZnO}$ QDs.

Inset Figure 2a shows the amorphous nature of graphene sheets and single crystalline structure of ZnO QDs by unresolved SAED pattern of the former and cubic SAED pattern of the latter, respectively.

\section{Figure 2}

Figure $2 b$ shows HRTEM image of ZnO QDs, being decorated on the graphene nanosheets. Measured lattice spacing of $\mathrm{d}_{011}=0.28 \mathrm{~nm}[40]$ and $\mathrm{d}_{002}=0.34 \mathrm{~nm}$ are consistent with the interplanar spacing of $\mathrm{ZnO}$ and graphene, respectively. Inset Figure 2b exhibits HR-TEM image of single $\mathrm{ZnO}$ QDs in which interplanar spacing in the crystal petals is $0.28 \mathrm{~nm}$ which corresponds to the distance between two (011) planes of hexagonal $\mathrm{ZnO}$ phase. Figure 2c demonstrates $\mathrm{ZnO}$-Graphene quasicore shell QDs in which hexagonal lattice of graphene shown in high magnification image.

3.2. Physical and Chemical Characterization: The X-ray diffraction (XRD) patterns of the GO, graphite, and $\mathrm{ZnO}-$ Graphene are depicted in the Figure S3. The XRD peak of GO shifted toward lower angles relative to the graphite as a result of intercalation of water and oxygen functionalities between graphitic layers in the GO. All characteristic peaks of $\mathrm{ZnO}$ exist in the XRD pattern of ZnO-Graphene. Thus, it is turned out that $\mathrm{ZnO}$ QDs in their pure form are deposited on the graphene (JCPDS 36-1451). An interesting point regarding the XRD pattern of $\mathrm{ZnO}-$ Graphene is absence of any peaks of GO or graphite in it. This phenomenon can be explained by considering the fact that in preparation of the GO specimens for XRD experiments, some sort of stacking of the layers takes place that brings about the GO peaks; whereas in $\mathrm{ZnO}-\mathrm{Graphene}$, the $\mathrm{ZnO}$ particles prevent restacking of the GO layers.

Raman spectroscopy was exploited to identify 1: Type of the carbon hybridization, 2: The degree of lattice disorder, and 3: Average domain size of hexagonal $\mathrm{sp}^{2}$ ring in the graphite-like materials[41]. The $\mathrm{I}_{\mathrm{D}} / \mathrm{I}_{\mathrm{G}}$ ratio in Raman spectra indicates the amount of defects and disorders that 
are correlated to the $\mathrm{sp}^{2} / \mathrm{sp}^{3}$ ratio. As shown in the Figure $3 \mathrm{a}, \mathrm{I}_{\mathrm{D}} / \mathrm{I}_{\mathrm{G}}$ ratio in $\mathrm{GO}$ is higher $\left(\mathrm{I}_{\mathrm{D}} / \mathrm{I}_{\mathrm{G}}\right.$ $=1.52$ ) than graphite, because oxidation process during Hummers method increases the size of $\mathrm{sp}^{3}$ domain. However, this ratio decreased noticeably in $\mathrm{ZnO}-$ Graphene implying that anchoring of $\mathrm{ZnO}$ QDs on the surface of graphene decreased the $\mathrm{sp}^{3} / \mathrm{sp}^{2}$ ratio. To further investigate the structural aspects of the materials, the experimental data were fitted by Gaussian fitting curves for identification of the peak positions and full width of half-maxima (FWHM) of D, G, and 2D bands (Figure S4a,b, and c in supplementary information). The FWHM of the D band was used as an indicator of the degree of the crystallinity. In this regard, the FWHM of the D band for graphite and GO were found to be 86 and $107\left(\mathrm{~cm}^{-1}\right)$, respectively; whereas for the $\mathrm{ZnO}$-Graphene it was reduced to $63 \mathrm{~cm}^{-1}$. This attenuation indicates that grafting of ZnO QDs on the surface of graphene using C-O-Zn bonds decreases the degree of crystallinity of the graphene, an effect that was previously observed in Figure 3a, in which the characteristic peaks of GO and graphite disappeared in the XRD pattern of $\mathrm{ZnO}-$ Graphene (see discussion of the previous paragraphs). Apparently, we are facing with two contradictive phenomena as follow

1: The $\mathrm{sp}^{3} / \mathrm{sp}^{2}$ ratio obeys the following order: $\quad \mathrm{GO}>\mathrm{ZnO}$-Graphene $>$ Graphite

2: Crystallinity represents a different arrangement: Graphite $>\mathrm{GO}>\mathrm{ZnO}$-Graphene

If one accepts that during deposition of $\mathrm{ZnO}$ QDs on GO, most of the oxygen functionality of the GO are removed, the reason for decreasing $\mathrm{sp}^{3} / \mathrm{sp}^{2}$ ratio in $\mathrm{ZnO}-\mathrm{Graphene}$ is found. On the other hand, our perception of the crystallinity is closely related to: stacking of graphene layers for forming a multilayered materials. Thus, the material with fewer layers presents lesser crystallinity. Since $\mathrm{ZnO}$ QDs prevent pill up of the graphene layers even in the solid form (i.e. specimen for Raman or XRD tests), the number of layers and hence crystallinity of $\mathrm{ZnO}$-Graphene is minimum in the above series.

\section{Figure 3}

Thermogravimetric (TG) analysis was utilized to study thermal stability of the prepared materials as well as of the evaluating of their $\mathrm{ZnO}$ and graphene contents. The TG curve (Figure 
3b) illustrates three major weight losses for the GO. The first one, an $8 \%$ weight loss up to $110{ }^{\circ} \mathrm{C}$ corresponds to the absorbed water on the GO nanosheets. The second weight loss at $225^{\circ} \mathrm{C}(30 \%)$ is attributed to the loss of $\mathrm{CO}$ and $\mathrm{CO}_{2}$ from the decomposition of the oxygen-containing functional groups. The third and the main weight loss (50\%) of GO refers to oxidation of carbon atoms at high temperature and in the presence of air. The TG curve of RGO demonstrates just one major weight loss $(65 \%)$ which is attributed to the oxidation of carbon atoms. In the case of $\mathrm{ZnO}$ QDs, a single weight loss occurs around $250{ }^{\circ} \mathrm{C}$ which corresponds to removing of the volatile organic functional groups from surface of the $\mathrm{ZnO}$ QDs. The $\mathrm{ZnO}-$ Graphene hybrid with different graphene content depict all of the above-mentioned weight losses including those corresponding to oxygencontaining functional groups, volatile functional groups, and carbon oxidation. Measurement of carbon oxidation in the $\mathrm{ZnO}$-Graphene hybrid is an effective method to evaluate the carbon content

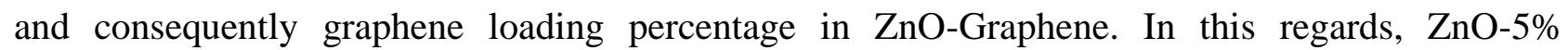
Graphene, $\mathrm{ZnO}-10 \%$ Graphene, and $\mathrm{ZnO}-30 \%$ Graphene in this study denote $\mathrm{ZnO}$-Graphene hybrid with 5, 10, and $30 \%$ of graphene loading, respectively. Unless otherwise stated, $\mathrm{ZnO}$ Graphene refers to $\mathrm{ZnO}-5 \%$ Graphene in this paper.

The FTIR spectra of GO and $\mathrm{ZnO}-$ Graphene are shown in Figure 3c. The successful oxidation of graphite in Hummer's method is affirmed by existence of different oxygen signatures in FTIR spectrum of the GO. In the case of $\mathrm{ZnO}-G r a p h e n e$, most of the oxygen functionalities were removed except $\mathrm{Zn}-\mathrm{O}$ stretching peak at $470 \mathrm{~cm}^{-1}$ and $\mathrm{C}-\mathrm{O}$ vibration one at $1400 \mathrm{~cm}^{-1}$. The aforementioned $\mathrm{Zn}-\mathrm{O}$ peak was kept because only one crystal layer in the bottom of $\mathrm{ZnO} \mathrm{NP}$ is connected to the graphene surface and the other (i.e. upper) layers can hold the $\mathrm{ZnO}$ crystal structure. The C-O peak on the other hand was kept because it was involved in linking of $\mathrm{Zn}$ to the GO through C-O-Zn bonding [29].

The chemical state and surface composition of GO and ZnO-Graphene were investigated by XPS analysis. For this purpose, the binding energies obtained by XPS analysis were standardized using carbon peak at $284.8 \mathrm{eV}$ as a reference. Full scan survey of $\mathrm{GO}$ and $\mathrm{ZnO}$-Graphene 
demonstrates main characteristic peaks of $\mathrm{C}, \mathrm{O}$, and $\mathrm{Zn}$ apart from $\mathrm{O}$ and $\mathrm{Zn}$ auger peaks in Figure $3 d[42]$.

In order to better survey the effect of $\mathrm{ZnO}$ deposition on the deoxygenation of $\mathrm{GO}$, a comparison was made between deconvoluted C1s XPS spectra of the GO and ZnO-Graphene. The binding energy locations and surface area under the curves for each peak are assigned in the Figures $4 \mathrm{a}$ and $\mathrm{b}$. As it can be seen, there are two obvious differences between the $\mathrm{C} 1 \mathrm{~s}$ peaks of GO and $\mathrm{ZnO}-$ Graphene. First, higher $\mathrm{C} / \mathrm{O}$ ratio of $\mathrm{ZnO}-$ Graphene compared to the $\mathrm{GO}$, due to the reduction of GO as a result of grafting of $\mathrm{ZnO}$ QDs on the surface of GO (Table 1), and second, emerging of C-Zn, C-O-Zn, and pi-pi peaks in ZnO-Graphene hybrids implying that ZnO QDs are bonded to the surface of graphene sheets.

In order to quantitatively evaluate the surface concentrations of carbon-bonded functional groups, the peak area of oxygen-containing bonds and $\mathrm{C} / \mathrm{O}$ ratio were calculated and inserted in Table 1. The relative concentration of the oxygen functional groups on the surface of $\mathrm{ZnO}$ Graphene is lowered by about $40 \%$ in comparison to pristine GO.

\section{Figure 4}

\section{Table 1}

3.3. Connection mechanism: Comprehending of detailed formation mechanism of the ZnO QDs on the surface of GO, and recognizing of the nature of chemical bonds between GO and ZnO QDs are difficult tasks. In an effort for embarking on these issues, Son et al. suggested that ZnO QDs are connected to the surface of graphene through C-O-Zn bonding[33]. However, they didn't provide a supportive evidence for their suggestion. Although, strong connection of $\mathrm{C}-\mathrm{O}-\mathrm{Zn}$ bonds between flower-like three-dimension (3D) microstructures of $\mathrm{ZnO}$ and reduced graphene oxide reported[15], however, there are several differences between this and Song et al. such as synthesis method, $\mathrm{ZnO}$ structure and the size nanostructure. The current study is to deal with the above-mentioned issues by means of O1s, and Zn2p XPS peaks fitting by component Gaussian. The XPS peaks corresponding to $\mathrm{Zn}(2 \mathrm{p})$ core level of $\mathrm{ZnO}$ QDs is shown in Figure S5 in supplementary 
information, in which Zn2p energy level is divided into two distinct levels of $\mathrm{Zn} 2 \mathrm{p}_{3 / 2}$ at $1023 \mathrm{eV}$ and $\mathrm{Zn} 2 \mathrm{p}_{1 / 2}$ at $1046 \mathrm{eV}[43]$. As it can be seen, the energy splitting is around $23 \mathrm{eV}$, which corresponds to spin-orbit coupling and is within the standard reference value of $\mathrm{ZnO}$. It must be noticed that the peak position of the $\mathrm{Zn}(2 \mathrm{p})$ core level in $\mathrm{ZnO}-$ Graphene composites was shifted to higher binding energy $(1.2 \mathrm{eV})$ indicating the presence of new chemical bonds between $\mathrm{ZnO}$ QDs and carbon or oxygen functionalities. To further elucidate the issue, $\mathrm{Zn} 2 \mathrm{p}_{3 / 2}$ was fitted and embedded in Figure S6 with two component Gaussians at around $1022 \mathrm{eV}$ and $1022.8 \mathrm{eV}$ that correspond to C-O-Zn and $\mathrm{Zn}-\mathrm{O}$, respectively.

\section{Figure 5}

In addition, we exploited O1s peak as a reactive site on the graphene surface, to shed more light on the connection mechanism. The broad and low intensity O1s peak of ZnO-Graphene relative to GO is possibly attributed to the change in the number of chemical bonds of oxygen atoms in $\mathrm{ZnO}-G r a p h e n e$ [43]. Indeed, due to some sort of chemical interaction (bonding) between $\mathrm{Zn}^{2+}$ and oxygen-containing groups on the surface of GO, the full width at half maximum (FWHM) of O1s peak is enlarged in $\mathrm{ZnO}-G r a p h e n e$ [44]. To prove the presence of new chemical bonds in ZnO-Graphene composites, O1s spectra of GO and $\mathrm{ZnO}-$ Graphene are deconvoluted and shown in Figure 5a, and b. In Figure 5a, the O1s peak of GO includes two spectral peak appeared at 531.7 and $532.8 \mathrm{eV}$, attributed to $\mathrm{C}=\mathrm{O}$ (Carbonyl and Carboxyl) and $\mathrm{C}-\mathrm{O}$ (Epoxy and Hydroxyl) respectively [45]. The O1s spectra of $\mathrm{ZnO}-$ Graphene are fitted to four component Gaussians at 529, 530.5, 531.7, and 532.8 (Figure 5b). The emerging peaks at 529 and $530.5 \mathrm{eV}$ are attributed to $\mathrm{Zn}$ $\mathrm{O}$ and $\mathrm{C}-\mathrm{O}-\mathrm{Zn}$ bonds respectively.

With the information obtained from curve fitting of $\mathrm{C} 1 \mathrm{~s}, \mathrm{O} 1 \mathrm{~s}$, and $\mathrm{Zn} 2 \mathrm{p}$ in hand, we are now able to put together a qualitative picture of the connection mechanism of $\mathrm{ZnO}$ QDs to the surface of graphene. The existence of different dangling bonds or functional groups on the surface of GO such as $\mathrm{COOH}, \mathrm{C}-\mathrm{OH}$, and $\mathrm{C}-\mathrm{O}-\mathrm{C}$, act as good nucleation sites to uniformly deposit $\mathrm{ZnO}$ QDs on the surface of graphene sheet. These functional groups immobilize $\mathrm{Zn}^{2+}$ ions through C-O- 
$\mathrm{Zn}$ bonds, thereby through condensation reaction, remaining $\mathrm{Zn}^{2+}$ can be connected to these nucleation sites and $\mathrm{ZnO}$ QDs grow on these sub-layers via normal ionic bonds.

Figure $5 \mathrm{c}$ is a schematic illustration demonstrating how oxygen-containing groups of GO provide nucleation sites for $\mathrm{ZnO}$ QDs to link them to the surface of graphene. As it can be noticed, $\mathrm{Zn}^{2+}$ in the form of $\mathrm{Zn}(\mathrm{OH})_{2}$ is connected via a ring opening mechanism by epoxy group and condensation mechanism to carboxyl and hydroxyl groups on the surface of GO[33].

3.4. Chemical stability of the prepared catalysts: Due to instability of zinc oxide in acidic media, it is important to evaluate the stability of the $\mathrm{ZnO}$ QDS and $\mathrm{ZnO}-$ Graphene hybrid in $\mathrm{pHs}<7$, where it is supposed to be utilized. Dissolution of $\mathrm{ZnO}$ in acids is presented by the following reactions:

$$
\begin{aligned}
& \mathrm{ZnO}_{(s)}+2 \mathrm{H}_{(a q)}^{+} \longleftrightarrow \mathrm{Zn}_{(a q)}^{2+}+\mathrm{H}_{2} \mathrm{O}_{(l)} \\
& \mathrm{ZnO}_{(s)}+\mathrm{H}_{(a q)}^{+} \longleftrightarrow \mathrm{Zn}(\mathrm{OH})_{(a q)}^{+}
\end{aligned}
$$

The $\mathrm{pH}$ of the solution plays a major role in the extent and velocity of the $\mathrm{ZnO}$ QDs dissolution. In order to elucidate such effects, three different quantum dots including bare $\mathrm{ZnO}$, $\mathrm{ZnO}-5 \%$ Graphene, and $\mathrm{ZnO}-30 \%$ Graphene were selected and kinetics of their dissolution at $\mathrm{pH}=$ 1.0, 3.0, and 6.0 were studied by measurement of the $\mathrm{Zn}^{2+}$ concentration. Figure $6 \mathrm{a}$ and $\mathrm{S} 7$ show the dissolution rate of $\mathrm{ZnO}$ QDS at $\mathrm{pH} 1.0$ in which the equilibrium was attained at $\mathrm{t} \approx 4 \mathrm{~h}$. For $\mathrm{ZnO}$ QDS at $\mathrm{pH} 1.0$, the release of $\mathrm{Zn}^{2+}$ ions was around $73 \%$ of the theoretical mass. This is in agreement with the TG analysis in which around $25 \%$ of the ZnO QDs comprised of the volatile surface functional groups. Consequently, it can be concluded that at $\mathrm{pH} 1.0$, there was a complete dissolution of the $\mathrm{ZnO}$ for the bare $\mathrm{ZnO} \mathrm{QD}$. To elaborate the extent of dissolution of this QD at different $\mathrm{pHs}$, the UV-Vis absorbance of different solutions after reaching the equilibrium were measured and depicted in Figure 6b. As it can be seen, excitonic peak of ZnO QD at $365 \mathrm{~nm}$ disappeared at $\mathrm{pH} 1.0$, due to the complete dissolution of $\mathrm{ZnO}$ in such medium. The significantly higher stability of $\mathrm{ZnO}$ QDs at $\mathrm{pH} 3.0$ and pH 6.0 is evident in Figure 6b. The quantitative values 
of the dissolution rate at different $\mathrm{pHs}$ are inserted in Table 2. The bare $\mathrm{ZnO}$ QDs as seen are semistable at $\mathrm{pH} 3.0$, but quite stable at the higher $\mathrm{pH}$ (i.e. 6.0). Figure 6a shows dissolution rate of different samples normalized on the base of their $\mathrm{ZnO}$ contents. The applied solution had $\mathrm{pH}=1$. Since according to the $\mathrm{TG}$ analysis, $\mathrm{ZnO}$ content in $\mathrm{ZnO}-5 \%$ Graphene was around 95\%, this sample after dissolution of its $\mathrm{ZnO}$ content left $5 \%$ of graphene residue. However, for $\mathrm{ZnO}-30 \%$ Graphene dissolution was incomplete; most likely due to the partial wrapping of $\mathrm{ZnO}$ QDs by the graphene sheets. In other words, graphene as an impermeable surface provided a barrier against acids for the $\mathrm{ZnO}$ QDs. Similar behaviour was reported for PbS-Graphene composite in which around $40 \%$ of $\mathrm{PbS}$ QDs were remained un-contacted in strong acidic medium [28]. Further evidence for protection effect of graphene can be seen in the UV-Visible spectra of $\mathrm{ZnO}-30 \%$ Graphene at different pHs in Figure 6d. In contrast to bare $\mathrm{ZnO}$ QDs, ZnO-30\% Graphene shows the excitonic peak at $365 \mathrm{~nm}$ at $\mathrm{pH}$ 1.0, implying that higher percentage of graphene results in higher stability of ZnO QDs at acidic medium. Figure S8 in supplementary information depicts the $\mathrm{UV}-\mathrm{V}$ is spectra of $\mathrm{ZnO}-10 \%$ Graphene at different $\mathrm{pH}$ values.

\section{Figure 6}

\section{Table 2}

3.5. Precipitation behaviour of the prepared catalysts. The precipitation stability of the synthesized catalysts in DMF and water was examined using time-dependent sedimentation curves (Figure 7). For the bare $\mathrm{ZnO}$ QDs dispersion is highly stable because surface organic functional groups act as surfactant, and the $\mathrm{A} / \mathrm{A}_{0}$ ratio ( $\mathrm{A}$, extinction at time $\mathrm{t} ; \mathrm{A}_{0}$, extinction at time $\mathrm{t}=0$ ) is nearly constant, with a slight descending slope. Replacing $\mathrm{ZnO}$ with graphene in the $\mathrm{ZnO}$ Graphene composite increases the precipitation tendency of the hybrid, as a result of aggregation of graphene sheets with each other. In this respect, it is expected that $\mathrm{ZnO}-30 \%$ Graphene shows a faster sedimentation rate than the $\mathrm{ZnO}-5 \%$ Graphene (Figure 7). The affinity of graphene sheets for aggregation is arisen from the $\pi-\pi$ interaction between the adjacent plans. 


\section{Figure 7}

3.6. Photocatalytic Properties: The UV-visible absorption spectra of $\mathrm{ZnO}$ QDs and ZnOGraphene hybrid with different graphene ratios are shown in Figure 8a. The $372 \mathrm{~nm}$ absorption excitation peak of $\mathrm{ZnO}$ QDs is corresponding to $3.15 \mathrm{eV}$ band gap. It can be observed that introducing of graphene sheet not only increases intensity of light absorption in the UV region, but also extends the light absorption range into visible area. Figure $8 \mathrm{~b}$ exhibits a plot of the transformed Kubelka-Munk function as a function of energy of photons, by which the estimated band gaps were found 2.7, 2.8, 2.9, and $3.15 \mathrm{eV}$ for $\mathrm{ZnO}-30 \%$ Graphene, $\mathrm{ZnO}-10 \%$ Graphene, $\mathrm{ZnO}-5 \%$ Graphene, and bare $\mathrm{ZnO}$ QDs, respectively. This red shift in the absorption edge of $\mathrm{ZnO}$-Graphene hybrid and consequently visible light activation can be attributed to the chemical bonding between ZnO QDs and the active oxygen-bearing nucleation sites on the surface of GO. In other words, the formation of $\mathrm{Zn}-\mathrm{O}-\mathrm{C}$ bonds between $\mathrm{ZnO}$ QDs and graphene as was discussed in the "connection mechanism" section, involves the band gap narrowing of $\mathrm{ZnO}$ QDs, and broadens the light absorption range of $\mathrm{ZnO}$ QDs toward the visible region (Figure $8 \mathrm{~b}$ ). The visible light activation of graphene-based semiconductors due to the chemical connection was reported previously [46-49].

\section{Figure 8}

The time-dependent UV-VIS spectra of MO degradation under UV light irradiation are illustrated in Figure S9 in supporting information file. As it can be noticed, the absorbance peak of $\mathrm{MO}$ at $464 \mathrm{~nm}$ entirely attenuates in $30 \mathrm{~min}$. Figure $9 \mathrm{a}$, and b show the degradation rate of MO under UV and visible light irradiation for the bare ZnO QDs and the hybrid QDs with different loading of graphene. Figure 9a shows the MO photo-degradation kinetics under UV irradiation fitted by the first-order kinetics model:

$\operatorname{Ln}\left(\frac{C}{C_{0}}\right)=-k t \quad$ or $\quad C=C_{0} e^{-k t}$ 
In the absence of catalyst, there is no degradation of the dye in the solution. The photocatalytic efficiency of QDs under both UV and Visible irradiation follows the following order: $\mathrm{ZnO}-5 \%$ Graphene > ZnO-10\% Graphene > ZnO QDs > ZnO-30\% Graphene. The slope of the $\mathrm{ZnO}-5 \%$ Graphene curve is nearly two times greater than that of the $\mathrm{ZnO}$ QDs under UV light irradiation, implying two times faster kinetics at the same condition. In other word, slight introduction of graphene (i.e. 5\%) into $\mathrm{ZnO}$ catalyst, leads to significant enhancement of its photocatalytic activity. Interestingly, this effect is greater in the visible region, in which the bare $\mathrm{ZnO}$ QDs shows a rather low catalytic activity (see discussion below). As shown in Figure 9, in both UV and visible regions, increasing the loading of graphene to $10 \%$, and then $30 \%$, leads to a profound decrease of efficiency of photocatalyst, even lower than ZnO QDs. Probably, at high weight percentage of graphene, it will cover or shade $\mathrm{ZnO}$ QDs and decreases their photodegradation efficiency.

As it can be observed in Figure 9b, photocatalytic activity of the $\mathrm{ZnO}$ QD is attenuated remarkably under visible light, because its band gap excitation peak lies in the UV region ( $\lambda=365$ $\mathrm{nm}$ [43]. On the other hand, $\mathrm{ZnO}-5 \%$ Graphene hybrid shows superior photocatalytic activity under visible light region possibly because graphene sheet can: 1) Extend the light absorption of the

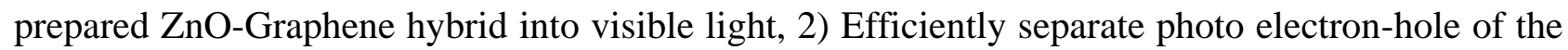
$\mathrm{ZnO}$ semiconductor, and reduce their recombination rate, and 3) Effectively adsorb the MO molecules and hold them in the vicinity of the $\mathrm{ZnO}$ QDs for decomposition. Between the aforementioned three mechanisms, the first one was discussed formerly, and the second will be addressed in the succeeding paragraphs, but the third one needs more clarification as follows. We investigated the adsorption ability of $\mathrm{ZnO}$ QDs and $\mathrm{ZnO}$-Graphene composite in dark according to the procedure given in the experimental section, and depicted the results in Figure S10 of supporting information. Evidently, adsorption of $\mathrm{MO}$ by $\mathrm{ZnO}$ was improved by anchoring of $\mathrm{ZnO}$ QDs on the surface of graphene as a result of both $\pi$ - $\pi$ interaction between MO molecules and graphene, and high surface area of the graphene sheets[50]. 


\section{Figure 9}

The light absorption, charge transportation, and separation of electron-hole are three important electronic mechanisms of the photocatalysis reaction. The charge transfer and electron-hole separation play significant role in both photocatalytic activity and photocorrosion effect. As it is shown in Figure S11 (Supporting information), ZnO QDs can be excited by UV light and provide electron-hole pairs in which the photo-induced electrons are excited from the valence band (VB) to conduction band (CB) forming a hole in the VB. Because the valence band (VB) position of $\mathrm{ZnO}$ QDs is lower than the highest occupied molecular orbital (HOMO) of graphene, the photo-induced holes could transfer to graphene[51]. Such hole transfer increases the lifetime of the free electrons of the $\mathrm{ZnO}$ QDs, and reduces their recombination rate with the holes, causing an enhanced level of photocatalytic activity.

Due to wide band gap (3.2 eV), $\mathrm{ZnO}$ QDs is not excited by visible light irradiation[52]. Because the conduction band (CB) location of $\mathrm{ZnO}$ QDs is lower than the low unoccupied molecular orbital (LUMO) of graphene, the photogenerated electrons by visible light could transfer from graphene sheets to the $\mathrm{CB}$ of $\mathrm{ZnO}$ QDs. These electrons, then transfer to the surface of photocatalyst and react with water and oxygen resulting in superoxide radicals production (see discussion of S11 figure in Supporting information file). The aforementioned explanation demonstrates that the mechanism of photocatalytic activation of $\mathrm{ZnO}$ by Graphene in the case of visible light is different with that of the UV irradiation.

3.7. Photocorrosion Suppression: One of the most important properties of the industrial photocatalysts is that their photocatalytic efficiency should be maintained in the successive cycles of degradation of organic compounds[53]. In this regards, the cyclic experiments for the degradation of $\mathrm{MO}$ on $\mathrm{ZnO}$ - graphene hybrids were conducted. As shown in Figure 10, under UV irradiation about $80 \%$ of $\mathrm{MO}$ could be degraded using $\mathrm{ZnO}$ QDs for the first time. As it can be seen, after three cycles of usage, a considerable reduction in photocatalytic activity of ZnO QDs 
was observed so that only $52 \%$ of $\mathrm{MO}$ could be decomposed, presumably due to the photocorrostion effect. However, when $\mathrm{ZnO}$ surface is covered-passivated by the graphene sheets, $\mathrm{ZnO}-$ Graphene composites represent long-lasting photostability. This can be observed in Figure 10 in which photocatalytic activity of $\mathrm{ZnO}-5 \%$ Graphene and $\mathrm{ZnO}-30 \%$ Graphene reduced merely $8 \%$ and $2 \%$, respectively, after three recycling experiments.. This photocorrosion inhabitation could be attributed to the close contact between $\mathrm{ZnO}$ QDs and graphene sheets through $\mathrm{Zn}-\mathrm{O}-\mathrm{C}$ and $\mathrm{Zn}-\mathrm{C}$ chemical bonds (See Formation Mechanism Section) which brings about to the graphene its protective effect. The higher photocorrosion suppression of $\mathrm{ZnO}-30 \%$ Graphene compared to $\mathrm{ZnO}$ $5 \%$ Graphene could be attributed to its higher graphene content. Similar to this results is presented in dissolution stability of $\mathrm{ZnO}-$ Graphene composites in which high graphene content protect $\mathrm{ZnO}$ QDs at low pH (Figure 6a).

\section{Figure 10}

To manifest the effect of photocorrosion phenomena, the absorption peak of $\mathrm{ZnO}$ QDs, and $\mathrm{ZnO}$ $30 \%$ Graphene before and after $24 \mathrm{hr}$ UV irradiation were obtained. As shown in Figure 11a and b, the absorption peak of $\mathrm{ZnO}$ QDs almost disappeared after $24 \mathrm{hr}$ photocatalytic activity due to photocorrostion effect, while the absorption peak of ZnO-30\% Graphene after $24 \mathrm{hr}$ UV irradiation is nearly identical to its zero irradiation level (Figure 11b). Furthermore, the photocorrostion inhabitation can be examined by measuring $\mathrm{Zn}^{2+}$ concentration before and after photocatalytic reaction. Table 3 listed $\mathrm{Zn}^{2+}$ ions concentration for $\mathrm{ZnO}$ QDs, $\mathrm{ZnO}-5 \%$ Graphene, and $\mathrm{ZnO}-30 \%$ Graphene indicating destruction of $\mathrm{ZnO}$ QDs crystal structure in the absence of graphene sheets and releasing more $\mathrm{Zn}^{2+}$ ions through the following reaction[53]:

$$
\mathrm{ZnO}+2 \mathrm{~h}^{+}+n \mathrm{H}_{2} \mathrm{O} \longrightarrow \mathrm{Zn}(\mathrm{OH})_{n}^{(2-n)+}+0.5 \mathrm{O}_{2}+n \mathrm{H}^{+}
$$

where $\mathrm{n}$ depends on the $\mathrm{pH}$ of the solution. The above mentioned reaction indicates that the photoinduced holes get involved in the photocorrostion of ZnO QDs crystal in aqueous solutions. 
As it was mentioned in the previous section, in $\mathrm{ZnO}$-graphene composite, holes are transferred from the $\mathrm{ZnO}$ QDs to the graphene sheets, and thus, they are no longer available to corrode the surface of $\mathrm{ZnO}$ via the reaction (5) mechanism. In other words, in the absence of water, the photocorrosion effect could be neglected. In this regards, the UV-visible absorbance of ZnO QDs and $\mathrm{ZnO}-30 \%$ Graphene in DMF solution are exhibited in Figure 11c and d, in which there is no difference between absorption peak before and after UV irradiation.

\section{Table 3}

\section{Figure 11}

4. Conclusions: In summary, we have for the first time tackled the deposition - formation mechanism of the ZnO QDs on the surface of GO which can be used for understanding of the immobilization of other metal oxides on the surface of GO. Integration of the ZnO QDs with graphene brings about three significant outcomes: 1- GO acts as a substrate for nucleation and growth of the $\mathrm{ZnO}$ QDs, and at the same time hinders their aggregation. In the absence of such substrate, $\mathrm{ZnO}$ QDs tend to agglomerate and produce larger particles. 2- Graphene adsorb the organic molecules and holds them near to $\mathrm{ZnO}$ QDs to be degraded by $\mathrm{ZnO}$ by different electronicchemical mechanisms and 3: The electron-hole pairs in the excited ZnO QDs can be efficiently separated through the transport of electrons or to the graphene. As a whole, existence of graphene improves the photocatalytic activity of the $\mathrm{ZnO}$ QDs and broaden the absorption range of wavelength toward visible light. Thus the prepared $\mathrm{ZnO}$-Graphene composites show promising photodegradation properties, especially in the visible region. Introduction of graphene sheets not only increases the light absorption of $\mathrm{ZnO}$-Graphene composites, but also produces an activated photocatalysts that via extending electron-hole life-time results in efficient oxidation- reduction reaction for degradation of organic pollutants. It has been demonstrated that $\mathrm{ZnO}$-Graphene composites possess antiphotocorrosion properties especially in high loading content of graphene sheets. 
The current study synthesized the $\mathrm{ZnO}$-Graphene composite with different loading of graphene, ranging from 5 to $30 \%$, and investigated their various physicochemical properties. Our study showed that although the higher loading of graphene improves acid resistance and precipitation stability of the prepared catalyst, the highest photocatalytic activity is resulted when the amount of the graphene added is slight and around 5\%. 
Notes: The authors declare no competing financial interest.

Acknowledgments: The authors would like to expresses their gratitude to Ms. Mahnaz separdar for her appreciable discussion on the mechanisms of formation. They are also thankful to Department of Energy Engineering of Sharif University of Technology for financial supports of this project.

*Supporting Information file is available: Raman fitted spectra, TEM images, EDX analysis, Full scan XPS, Upshifting of Zn2p core level peak, Spectral degradation of MO, Photocatalytic mechanism. 


\section{5- References}

[1] W. Viessman, M.J. Hammer, E.M. Perez, P.A. Chadik, Water supply and pollution control, Pearson Prentice Hall New Jersey, NJ, 2009.

[2] N. Ghaffour, T.M. Missimer, G.L. Amy, Technical review and evaluation of the economics of water desalination: current and future challenges for better water supply sustainability, Desalination, 309 (2013) 197-207.

[3] A. Pérez-González, A. Urtiaga, R. Ibáñez, I. Ortiz, State of the art and review on the treatment technologies of water reverse osmosis concentrates, Water research, 46 (2012) 267-283.

[4] J. Fang, H. Fan, G. Dong, A facile way to synthesize cost-effective ZnO nanorods with enhanced photocatalytic activity, Materials Letters, 120 (2014) 147-150.

[5] M.R. Hoffmann, S.T. Martin, W. Choi, D.W. Bahnemann, Environmental applications of semiconductor photocatalysis, Chem. Rev., 95 (1995) 69-96.

[6] J. Fang, H. Fan, Y. Ma, Z. Wang, Q. Chang, Surface defects control for ZnO nanorods synthesized by quenching and their anti-recombination in photocatalysis, Applied Surface Science, 332 (2015) 47-54.

[7] H. Yu, H. Fan, X. Wang, J. Wang, Synthesis and characterization of $\mathrm{ZnO}$ microstructures via microwaveassisted hydrothermal synthesis process, Optik-International Journal for Light and Electron Optics, 125 (2014) 1461-1464.

[8] R. Lv, X. Wang, W. Lv, Y. Xu, Y. Ge, H. He, G. Li, X. Wu, X. Li, Q. Li, Facile synthesis of ZnO nanorods grown on graphene sheets and its enhanced photocatalytic efficiency, Journal of Chemical Technology \& Biotechnology, (2014) n/a-n/a.

[9] S. Banerjee, S.C. Pillai, P. Falaras, K.E. O'Shea, J.A. Byrne, D.D. Dionysiou, New Insights into the Mechanism of Visible Light Photocatalysis, The Journal of Physical Chemistry Letters, 5 (2014) 2543-2554.

[10] C. Han, M.-Q. Yang, N. Zhang, Y.-J. Xu, Enhancing the visible light photocatalytic performance of ternary CdS-(graphene-Pd) nanocomposites via a facile interfacial mediator and co-catalyst strategy, Journal of Materials Chemistry A, (2014).

[11] Z. Liu, Z. Liu, T. Cui, J. Li, J. Zhang, T. Chen, X. Wang, X. Liang, Photocatalysis of two-dimensional honeycomb-like ZnO nanowalls on zeolite, Chemical Engineering Journal, 235 (2014) 257-263.

[12] F.F. de Brites-Nóbrega, A.N.B. Polo, A.M. Benedetti, M.M.D. Leão, V. Slusarski-Santana, N.R.C. Fernandes-Machado, Evaluation of photocatalytic activities of supported catalysts on NaX zeolite or activated charcoal, Journal of Hazardous Materials, 263, Part 1 (2013) 61-66.

[13] H. Tian, H. Fan, H. Guo, N. Song, Solution-based synthesis of $\mathrm{ZnO} /$ carbon nanostructures by chemical coupling for high performance gas sensors, Sensors and Actuators B: Chemical, 195 (2014) 147-150.

[14] X. Bai, L. Wang, R. Zong, Y. Lv, Y. Sun, Y. Zhu, Performance Enhancement of ZnO Photocatalyst via Synergic Effect of Surface Oxygen Defect and Graphene Hybridization, Langmuir, 29 (2013) 3097-3105.

[15] N. Song, H. Fan, H. Tian, Reduced graphene oxide/ZnO nanohybrids: Metallic Zn powder induced onestep synthesis for enhanced photocurrent and photocatalytic response, Applied Surface Science, 353 (2015) 580-587.

[16] N. Zhang, Y. Zhang, Y.-J. Xu, Recent progress on graphene-based photocatalysts: current status and future perspectives, Nanoscale, 4 (2012) 5792-5813.

[17] A.K. Geim, K.S. Novoselov, The rise of graphene, Nat Mater, 6 (2007) 183-191.

[18] M. Tayebi, A. Ramazani S.A, M.T. Hamed Mosavian, A. Tayyebi, LDPE/EVA/graphene nanocomposites with enhanced mechanical and gas permeability properties, Polymers for Advanced Technologies, 26 (2015) 1083-1090.

[19] S. Goenka, V. Sant, S. Sant, Graphene-based nanomaterials for drug delivery and tissue engineering, Journal of Controlled Release, 173 (2014) 75-88.

[20] A. Lipatov, A. Varezhnikov, P. Wilson, V. Sysoev, A. Kolmakov, A. Sinitskii, Highly selective gas sensor arrays based on thermally reduced graphene oxide, Nanoscale, 5 (2013) 5426-5434. 
[21] B.F. Machado, P. Serp, Graphene-based materials for catalysis, Catalysis Science \& Technology, 2 (2012) 54-75.

[22] M. Tavakoli, A. Tayyebi, A. Simchi, H. Aashuri, M. Outokesh, Z. Fan, Physicochemical properties of hybrid graphene-lead sulfide quantum dots prepared by supercritical ethanol, Journal of Nanoparticle Research, 17 (2015) 1-13.

[23] D.R. Dreyer, A.D. Todd, C.W. Bielawski, Harnessing the chemistry of graphene oxide, Chemical Society Reviews, 43 (2014) 5288-5301.

[24] K.A. Mkhoyan, A.W. Contryman, J. Silcox, D.A. Stewart, G. Eda, C. Mattevi, S. Miller, M. Chhowalla, Atomic and Electronic Structure of Graphene-Oxide, Nano Letters, 9 (2009) 1058-1063.

[25] Q. Xiang, J. Yu, M. Jaroniec, Graphene-based semiconductor photocatalysts, Chemical Society Reviews, 41 (2012) 782-796.

[26] M.-Q. Yang, N. Zhang, M. Pagliaro, Y.-J. Xu, Artificial photosynthesis over graphene-semiconductor composites. Are we getting better?, Chemical Society Reviews, 43 (2014) 8240-8254.

[27] A. Tayyebi, M. Outokesh, S. Moradi, A. Doram, Synthesis and characterization of ultrasound assisted "graphene oxide-magnetite" hybrid, and investigation of its adsorption properties for $\mathrm{Sr}(\mathrm{II})$ and $\mathrm{Co}(\mathrm{II})$ ions, Applied Surface Science, 353 (2015) 350-362.

[28] A. Tayyebi, M.M. Tavakoli, M. Outokesh, A. Shafiekhani, A. Simchi, Supercritical Synthesis and Characterization of Graphene-PbS Quantum Dots Composite with Enhanced Photovoltaic Properties, Industrial \& Engineering Chemistry Research, 54 (2015) 7382-7392.

[29] B. Li, H. Cao, ZnO@graphene composite with enhanced performance for the removal of dye from water, Journal of Materials Chemistry, 21 (2011) 3346-3349.

[30] T. Lv, L. Pan, X. Liu, Z. Sun, Enhanced photocatalytic degradation of methylene blue by ZnO-reduced graphene oxide-carbon nanotube composites synthesized via microwave-assisted reaction, Catalysis Science \& Technology, 2 (2012) 2297-2301.

[31] J. Jasieniak, M. Califano, S.E. Watkins, Size-Dependent Valence and Conduction Band-Edge Energies of Semiconductor Nanocrystals, ACS Nano, 5 (2011) 5888-5902.

[32] T.J. Jacobsson, T. Edvinsson, Antireflective coatings of $\mathrm{ZnO}$ quantum dots and their photocatalytic activity, RSC Advances, 2 (2012) 10298-10305.

[33] D.I. Son, B.W. Kwon, D.H. Park, W.-S. Seo, Y. Yi, B. Angadi, C.-L. Lee, W.K. Choi, Emissive ZnO-graphene quantum dots for white-light-emitting diodes, Nat Nano, 7 (2012) 465-471.

[34] M.-C. Hsiao, S.-H. Liao, M.-Y. Yen, P.-I. Liu, N.-W. Pu, C.-A. Wang, C.-C.M. Ma, Preparation of Covalently Functionalized Graphene Using Residual Oxygen-Containing Functional Groups, ACS Applied Materials \& Interfaces, 2 (2010) 3092-3099.

[35] X. Wang, S.M. Tabakman, H. Dai, Atomic Layer Deposition of Metal Oxides on Pristine and Functionalized Graphene, Journal of the American Chemical Society, 130 (2008) 8152-8153.

[36] Q. Ma, X. Zhu, D. Zhang, S. Liu, Graphene oxide - a surprisingly good nucleation seed and adhesion promotion agent for one-step ZnO lithography and optoelectronic applications, Journal of Materials Chemistry C, 2 (2014) 8956-8961.

[37] W.S. Hummers, R.E. Offeman, Preparation of Graphitic Oxide, Journal of the American Chemical Society, 80 (1958) 1339-1339.

[38] S. Moradi, O. Akhavan, A. Tayyebi, R. Rahighi, M. Mohammadzadeh, H.S. Rad, Magnetite/dextranfunctionalized graphene oxide nanosheets for in vivo positive contrast magnetic resonance imaging, RSC Advances, 5 (2015) 47529-47537.

[39] P.K. Ang, S. Wang, Q. Bao, J.T.L. Thong, K.P. Loh, High-Throughput Synthesis of Graphene by Intercalation-Exfoliation of Graphite Oxide and Study of Ionic Screening in Graphene Transistor, ACS Nano, 3 (2009) 3587-3594.

[40] S.F. Yu, C. Yuen, S.P. Lau, W.I. Park, G.-C. Yi, Random laser action in ZnO nanorod arrays embedded in ZnO epilayers, Applied Physics Letters, 84 (2004) 3241-3243. 
[41] S. Webster, R. Czerw, D. Carroll, M. Terrones, N. Grobert, Raman Study of Doped Multiwalled Carbon Nanotubes, in: APS Meeting Abstracts, 2002, pp. 25011.

[42] A. Nourmohammadi, R. Rahighi, O. Akhavan, A. Moshfegh, Graphene oxide sheets involved in vertically aligned zinc oxide nanowires for visible light photoinactivation of bacteria, Journal of Alloys and Compounds, 612 (2014) 380-385.

[43] O. Akhavan, Photocatalytic reduction of graphene oxides hybridized by ZnO nanoparticles in ethanol, Carbon, 49 (2011) 11-18.

[44] D. Rosenthal, M. Ruta, R. Schlögl, L. Kiwi-Minsker, Combined XPS and TPD study of oxygenfunctionalized carbon nanofibers grown on sintered metal fibers, Carbon, 48 (2010) 1835-1843.

[45] D. Yang, A. Velamakanni, G. Bozoklu, S. Park, M. Stoller, R.D. Piner, S. Stankovich, I. Jung, D.A. Field, C.A. Ventrice Jr, R.S. Ruoff, Chemical analysis of graphene oxide films after heat and chemical treatments by X-ray photoelectron and Micro-Raman spectroscopy, Carbon, 47 (2009) 145-152.

[46] H. Zhang, X. Lv, Y. Li, Y. Wang, J. Li, P25-graphene composite as a high performance photocatalyst, ACS nano, 4 (2009) 380-386.

[47] H.N. Tien, N.T. Khoa, S.H. Hahn, J.S. Chung, E.W. Shin, S.H. Hur, One-pot synthesis of a reduced graphene oxide-zinc oxide sphere composite and its use as a visible light photocatalyst, Chemical Engineering Journal, 229 (2013) 126-133.

[48] N. Zhang, M.-Q. Yang, S. Liu, Y. Sun, Y.-J. Xu, Waltzing with the Versatile Platform of Graphene to Synthesize Composite Photocatalysts, Chemical reviews, 115 (2015) 10307-10377.

[49] Y. Zhang, Z.-R. Tang, X. Fu, Y.-J. Xu, TiO2- graphene nanocomposites for gas-phase photocatalytic degradation of volatile aromatic pollutant: is $\mathrm{TiO}_{2}-$ graphene truly different from other TiO2- carbon composite materials?, ACS nano, 4 (2010) 7303-7314.

[50] J. Wang, T. Tsuzuki, B. Tang, X. Hou, L. Sun, X. Wang, Reduced Graphene Oxide/ZnO Composite: Reusable Adsorbent for Pollutant Management, ACS Applied Materials \& Interfaces, 4 (2012) 3084-3090.

[51] Y. Wang, R. Shi, J. Lin, Y. Zhu, Enhancement of photocurrent and photocatalytic activity of ZnO hybridized with graphite-like C 3 N 4, Energy \& Environmental Science, 4 (2011) 2922-2929.

[52] H. Zhang, R. Zong, Y. Zhu, Photocorrosion inhibition and photoactivity enhancement for zinc oxide via hybridization with monolayer polyaniline, The Journal of Physical Chemistry C, 113 (2009) 4605-4611.

[53] L. Zhang, H. Cheng, R. Zong, Y. Zhu, Photocorrosion suppression of ZnO nanoparticles via hybridization with graphite-like carbon and enhanced photocatalytic activity, The Journal of Physical Chemistry C, 113 (2009) 2368-2374. 


\section{Figure Captions}

Figure1: (a) A typical AFM image of GO sheet on mica and height profile analysis of marked-line, (b) TEM image of GO sheet, inset demonstrating SAED pattern of GO, and (c) UV-visible absorbance of GO solution (inset optical picture of GO solution)

Figure2: Low magnification TEM image of $\mathrm{ZnO}-$ Graphene hybrid, inset corresponding SAED patterns of $\mathrm{ZnO}$ QDs and graphene, (b) HR-TEM image of ZnO-Graphene, inset HR-TEM image of single crystal of $\mathrm{ZnO}$ QDs, and (c) HR-TEM image of ZnO-Graphene hybrid in which Graphene sheets covered $\mathrm{ZnO}$ QDs (Left)

Figure3: (a) Raman spectra of synthesized materials, (b), Thermogravimetric analysis of prepared materials, (c) FTIR patterns of GO and ZnO-Graphene, and (d) The full survey XPS spectra of GO and $\mathrm{ZnO}-$ Graphene hybrid.

Figure4: XPS curve fitting of $\mathrm{C} 1$ s spectra of (a) GO, and (b) ZnO-Graphene.

Figure5: XPS curve fitting of O1s spectra of (a) GO, (b) ZnO-Graphene, and (c) Schematic illustration of $\mathrm{Zn}^{2+}$ connection to oxygen functionalities on the surface of GO.

Figure6: (a) Time-dependent measurements of $\mathrm{ZnO}$ QDs and $\mathrm{ZnO}$-Graphene dissolution based on Normalized $\mathrm{ZnO}$ content at different $\mathrm{pH}$, (b) UV-visible absorbance of $\mathrm{ZnO}$ QDs solution after reaching to equilibrium, and (c) UV-visible absorbance of $\mathrm{ZnO}-30 \%$ Graphene solution after reaching to equilibrium (100 mg/L initial mass of ZnO QDs and ZnO Graphene )

Figure7: Sedimentation plot of $\mathrm{ZnO}$ QDs, and $\mathrm{ZnO}-$ Graphene hybrid in (a) DMF , and (b) Water ( $\mathrm{pH} 7$ )

Figure 8: UV-Visible absorbance of $\mathrm{ZnO}$ QDs and $\mathrm{ZnO}-$ Graphene, and (b) The plot of transformed Kubelka-Munk function versus the energy of light.

Figure 9: Kinetic of photocatalytic degradation of MO under (a) UV and (b) Visible light irradiation for $\mathrm{ZnO}$ QDs and $\mathrm{ZnO}-$ Graphene hybrid. 
Figure 10: The photostability of $\mathrm{ZnO}$ QDs and $\mathrm{ZnO}$-Graphene composites by investigation of photocatalytic activity under UV irradiation, $($ Red circle $=\mathrm{ZnO}$ QDs, Green triangle $=\mathrm{ZnO}-30 \%$ Graphene, Blue Star = ZnO-5\% Graphene).

Figure 11: UV-Visible absorbance of (a) ZnO QDs, (b) ZnO-30\% Graphene in aquous solution, (c) ZnO QDs, and (d) ZnO-30\% Graphene in DMF solution before and after 24 UV irradiation. 
Table 1: Surface concentration of carbon-bonded groups of GO and $\mathrm{ZnO-Graphene,} \mathrm{calculated} \mathrm{from} \mathrm{peak}$ areas of the C1s spectra.

\begin{tabular}{ccccccc}
\hline \multirow{2}{*}{ Sample } & \multicolumn{7}{c}{ Peaks Area Percentage } \\
\cline { 2 - 7 } GO & 0 & 57 & 0 & 33 & 10 & 2.3 \\
ZnO-5\% Graphene & 6 & 52 & 25 & 7 & 6 & 7.6 \\
\hline
\end{tabular}

Table 2: $\mathrm{Zn}^{2+}$ ions concentration at effluent solution of $\mathrm{ZnO}$ QDs and $\mathrm{ZnO}$-Graphene hybrid at different $\mathrm{pH}$.

\begin{tabular}{|c|c|c|c|c|}
\hline Type of Catalyst & $\begin{array}{c}\mathrm{pH}=1 \\
(\mathrm{mg} / \mathrm{L})\end{array}$ & $\begin{array}{c}\mathrm{pH}=3 \\
(\mathrm{mg} / \mathrm{L})\end{array}$ & $\begin{array}{c}\mathrm{pH}=6 \\
(\mathrm{mg} / \mathrm{L})\end{array}$ & $\begin{array}{r}\mathrm{pH}=7.2 \\
(\mathrm{mg} / \mathrm{L})\end{array}$ \\
\hline ZnO QDs & 74 & 23 & 3.1 & 0.0 \\
\hline ZnO-5\% Graphene & 69 & 19 & 0.3 & 0.0 \\
\hline ZnO-30\% Graphene & 34 & 5 & 0.15 & 0.0 \\
\hline
\end{tabular}


Table 3: The concentration of $\mathrm{Zn}^{2+}$ ions in the solution of $\mathrm{ZnO}$ QDs and $\mathrm{ZnO}-$ Graphene photocatalytic process before and after UV irradiation $(\mathrm{pH}=6.5)$.

\begin{tabular}{ccc}
\hline Type of Catalyst & $\begin{array}{c}\text { Before UV irradiation } \\
(\mathbf{m g} / \mathrm{L})\end{array}$ & $\begin{array}{c}\text { After } \mathbf{2 4} \text { hr UV irradiation } \\
(\mathbf{m g} / \mathrm{L})\end{array}$ \\
\hline ZnO QDs & 1.4 & 71 \\
$\mathbf{Z n O - 5 \%}$ Graphene & 0.2 & 2.9 \\
ZnO-30\% Graphene & 0.05 & 1.3 \\
\hline
\end{tabular}



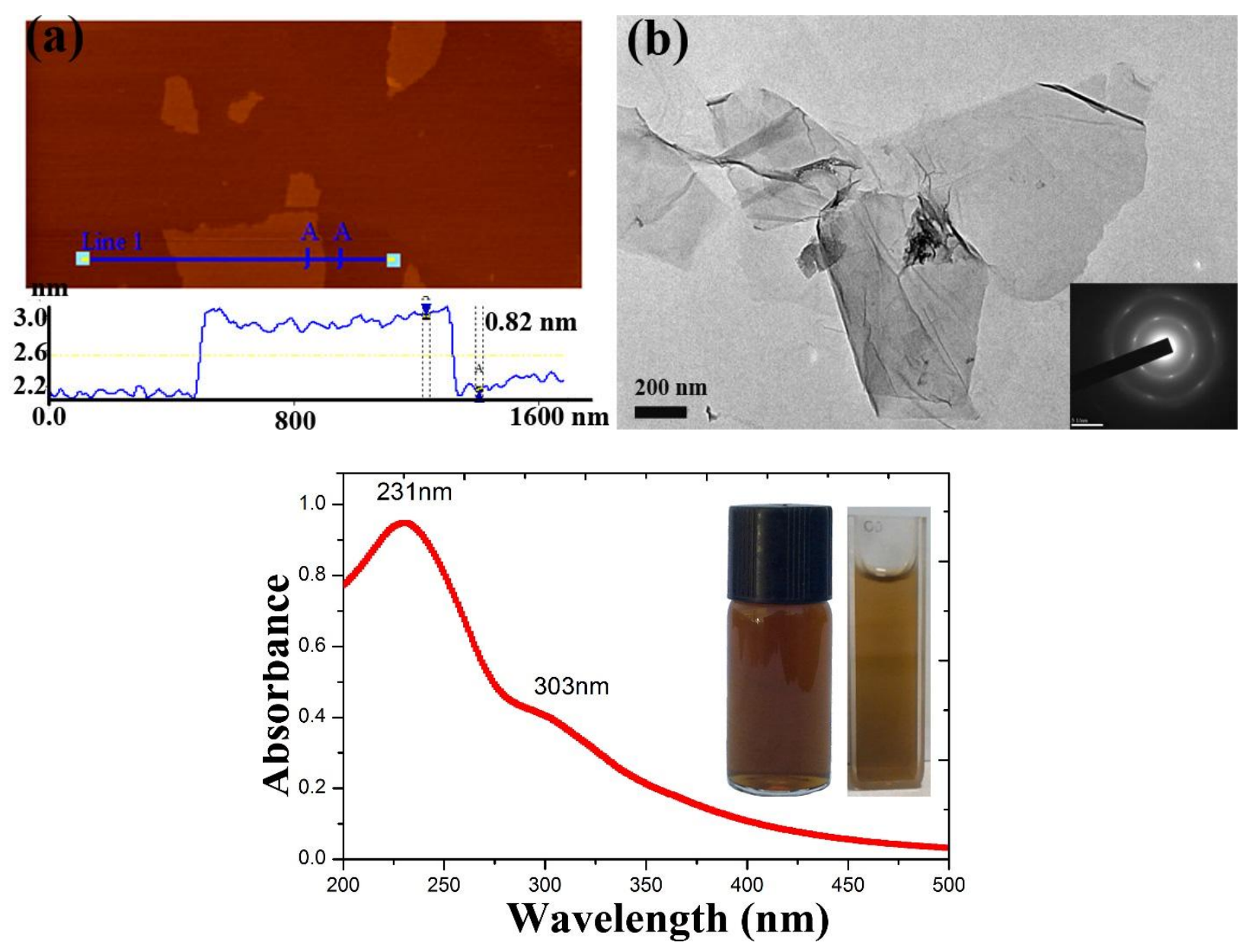

(c)

Figure1: (a) A typical AFM image of GO sheet on mica and height profile analysis of marked-line, (b) TEM image of GO sheet, inset demonstrating SAED pattern of GO, and (c) UV-visible absorbance of GO solution (inset optical picture of GO solution). 


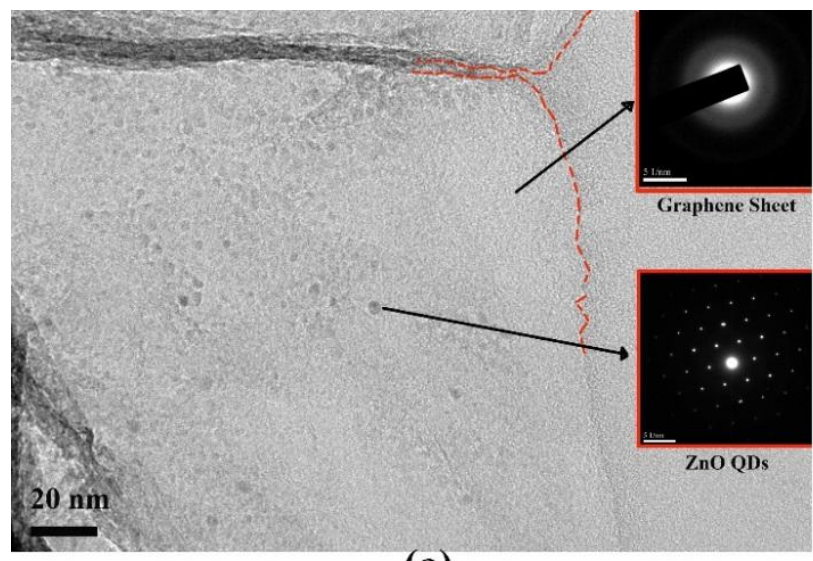

(a)

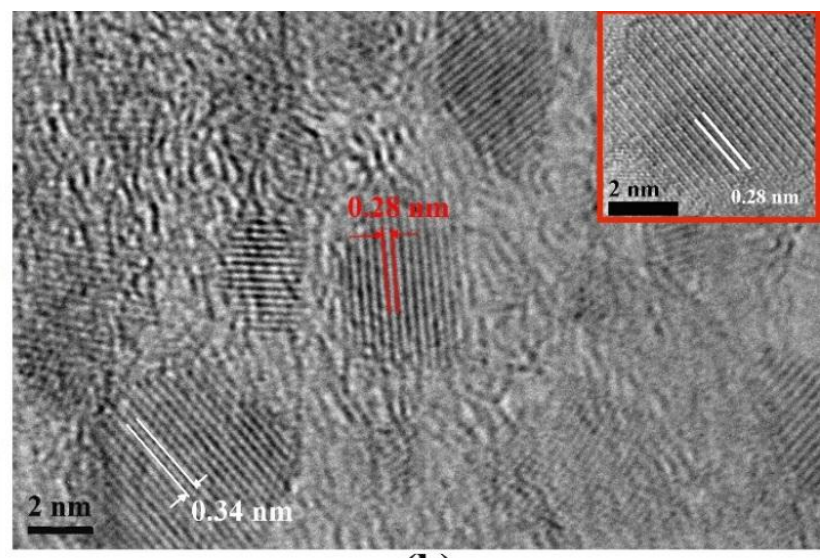

(b)
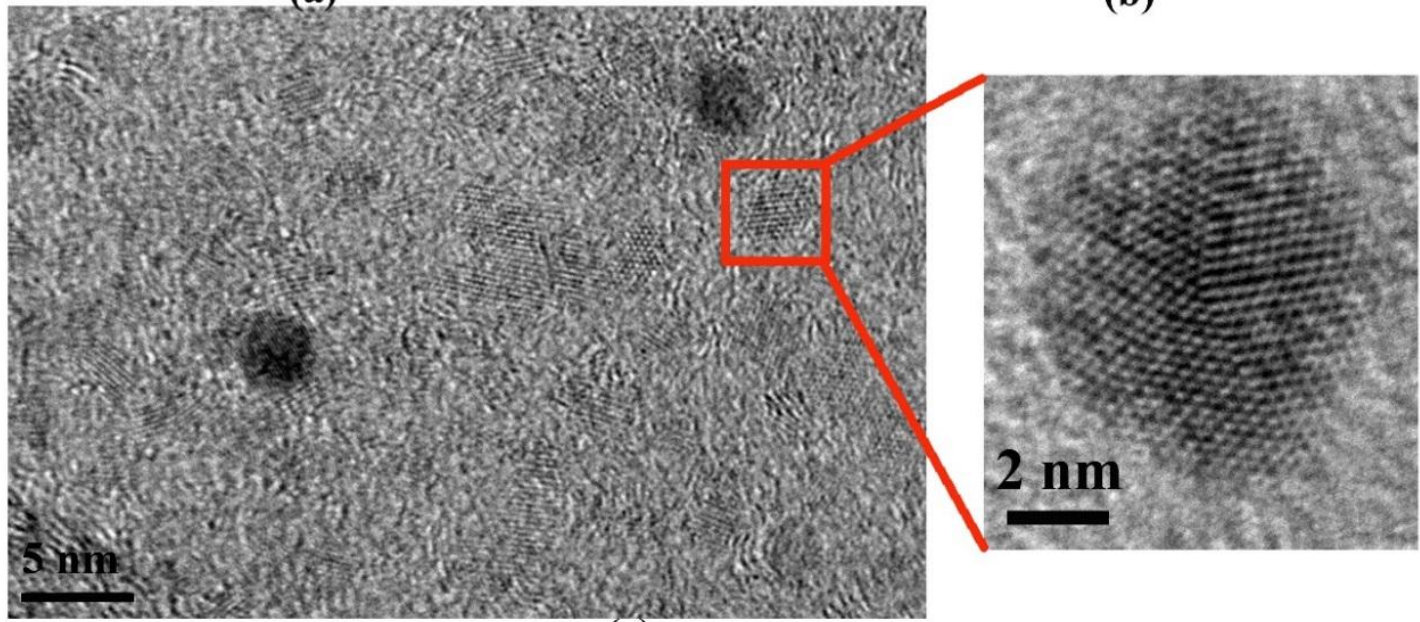

(c)

Figure2: Low magnification TEM image of ZnO-Graphene hybrid, inset corresponding SAED patterns of ZnO QDs and graphene, (b) HR-TEM image of ZnO-Graphene, inset HR-TEM image of single crystal of $\mathrm{ZnO}$ QDs, and (c) HR-TEM image of ZnO-Graphene hybrid in which Graphene sheets covered ZnO QDs (Left). 

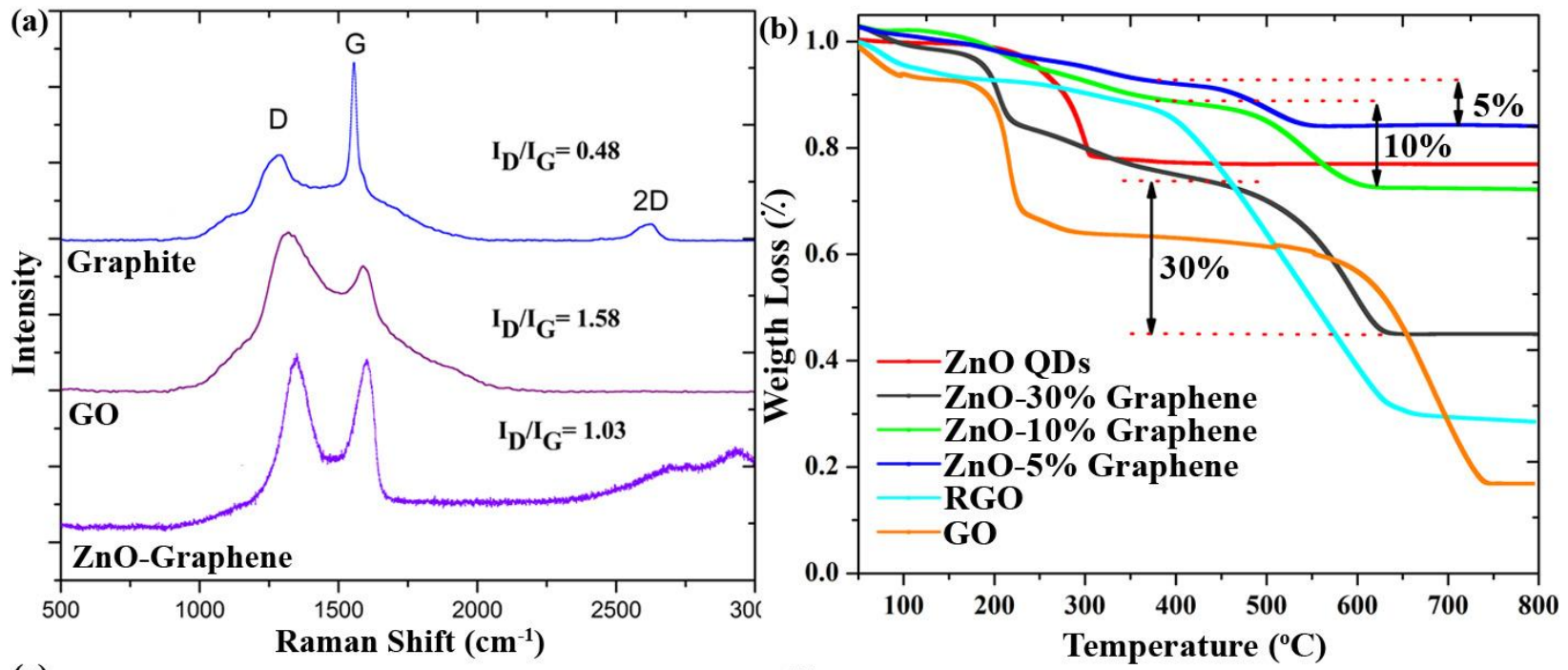

(c)

(d)
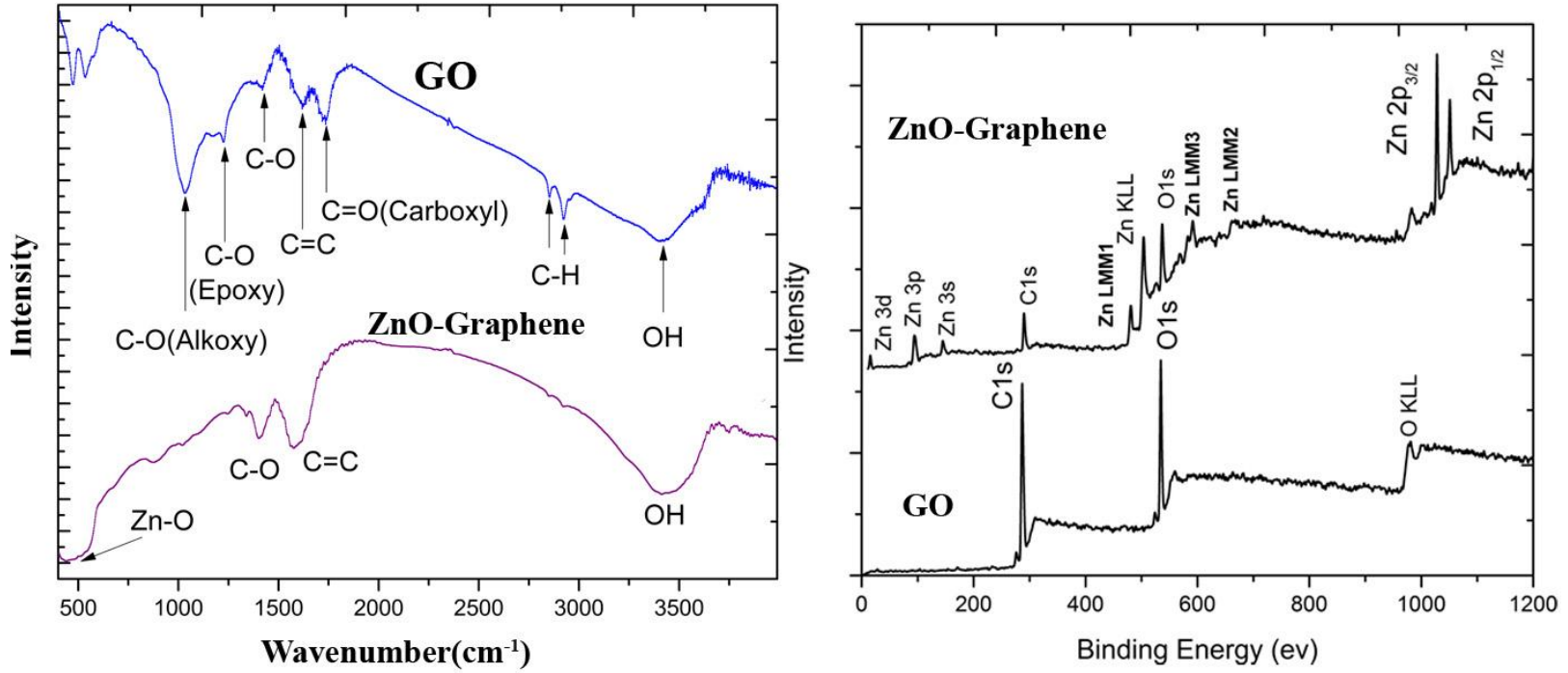

Figure3: (a) Raman spectra of synthesized materials, (b), Thermogravimetric analysis of prepared materials, (c) FTIR patterns of GO and ZnO-Graphene, and (d) The full survey XPS spectra of GO and $\mathrm{ZnO}-$ Graphene hybrid. 

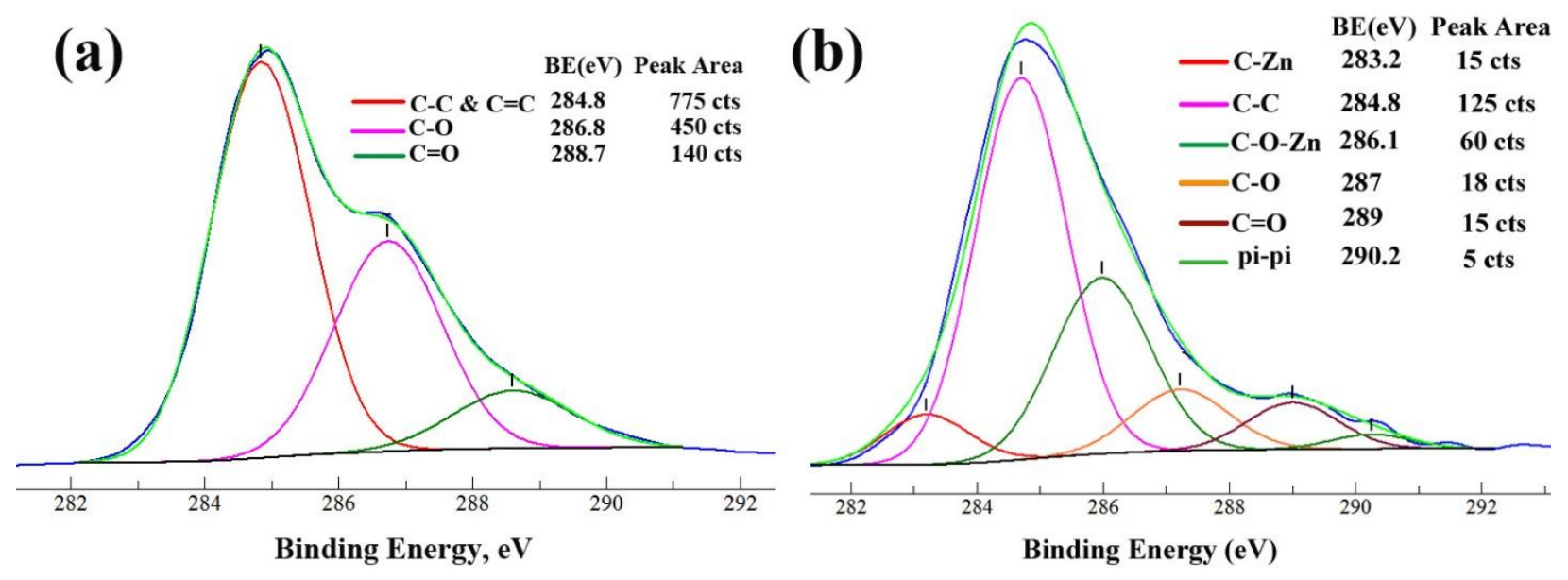

Figure4: XPS curve fitting of $\mathrm{C} 1$ s spectra of (a) GO, and (b) $\mathrm{ZnO}-\mathrm{Graphene}$. 


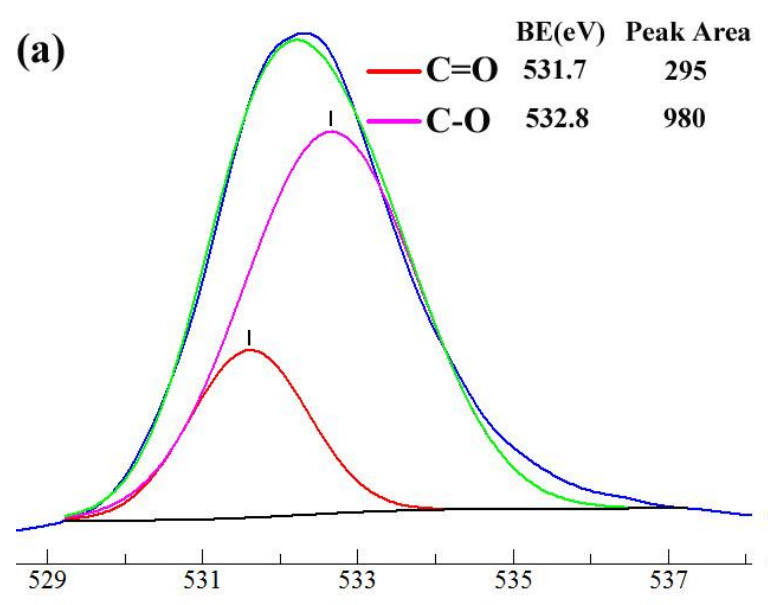

Binding Energy (eV)

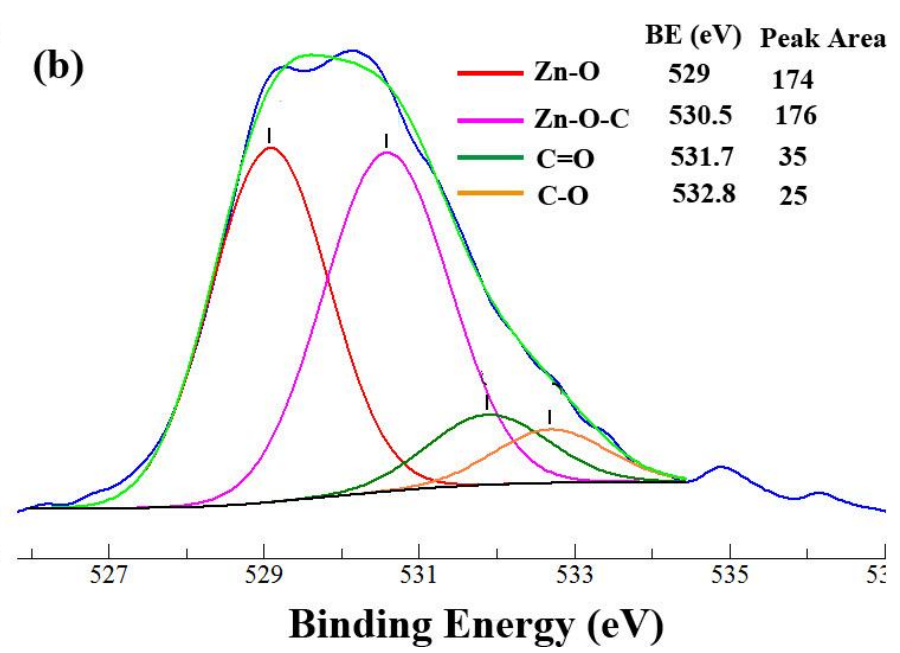

Binding Energy (eV)

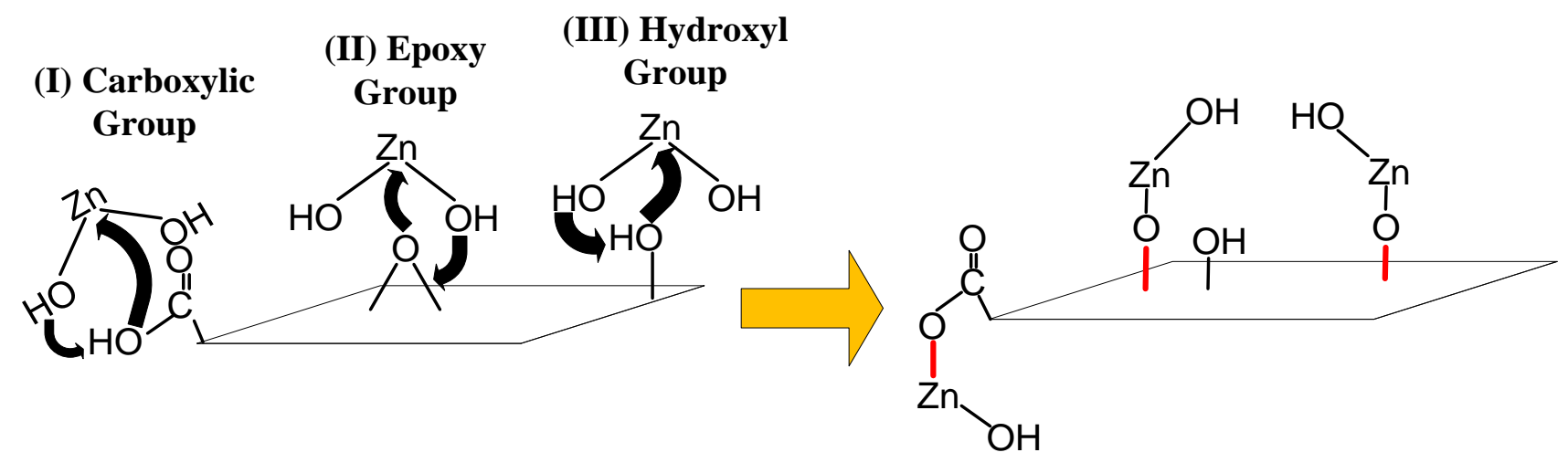

(c)

Figure5: XPS curve fitting of O1s spectra of (a) GO, (b) ZnO-Graphene, and (c) Schematic illustration of $\mathrm{Zn}^{2+}$ connection to oxygen functionalities on the surface of GO. 


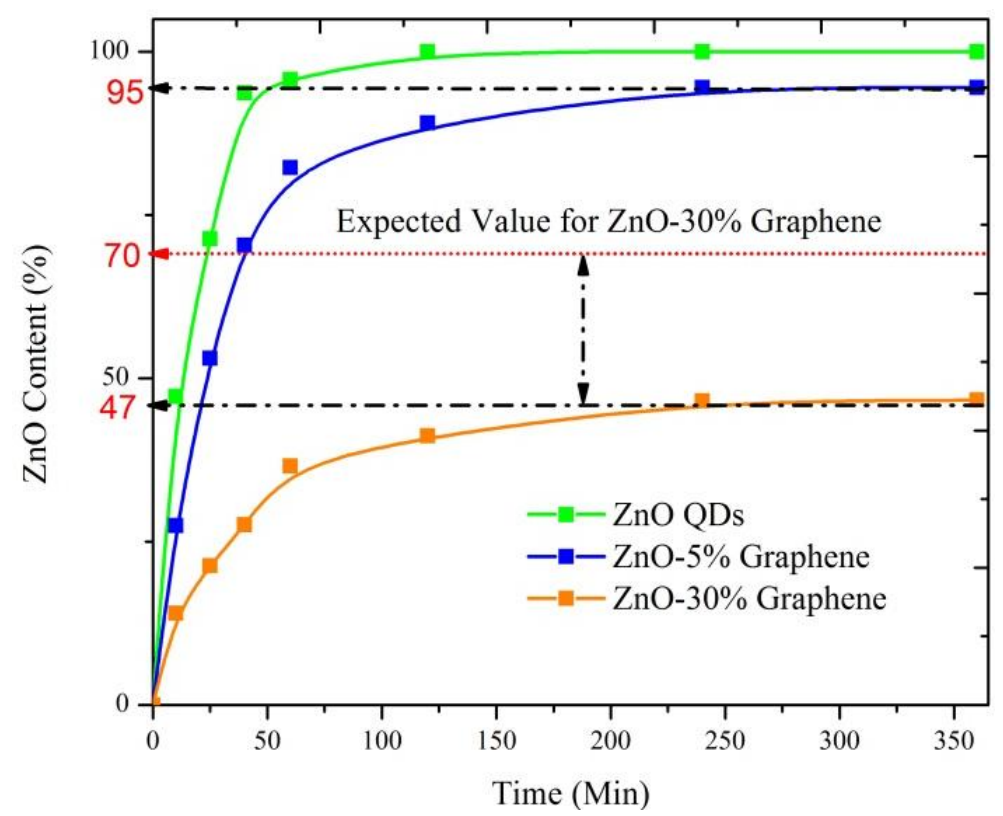

(a)

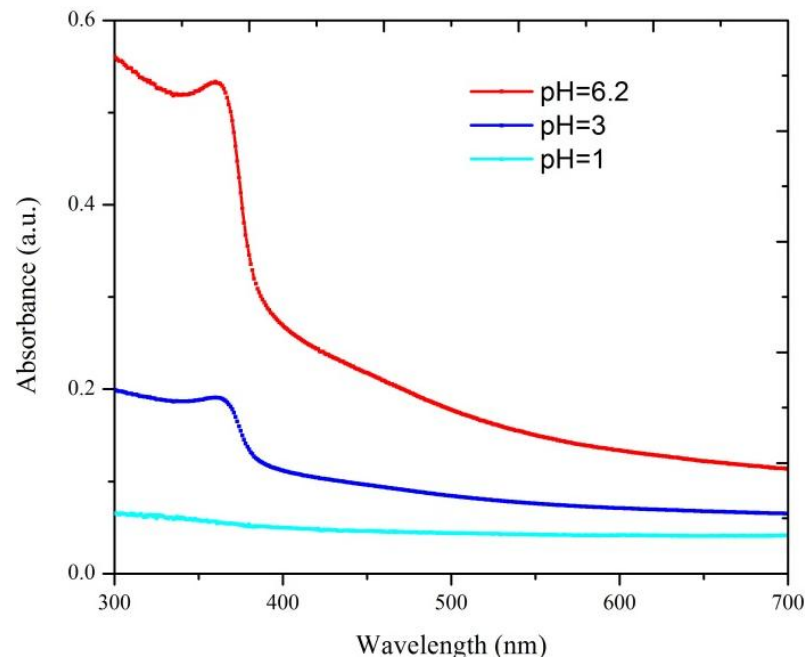

(b)

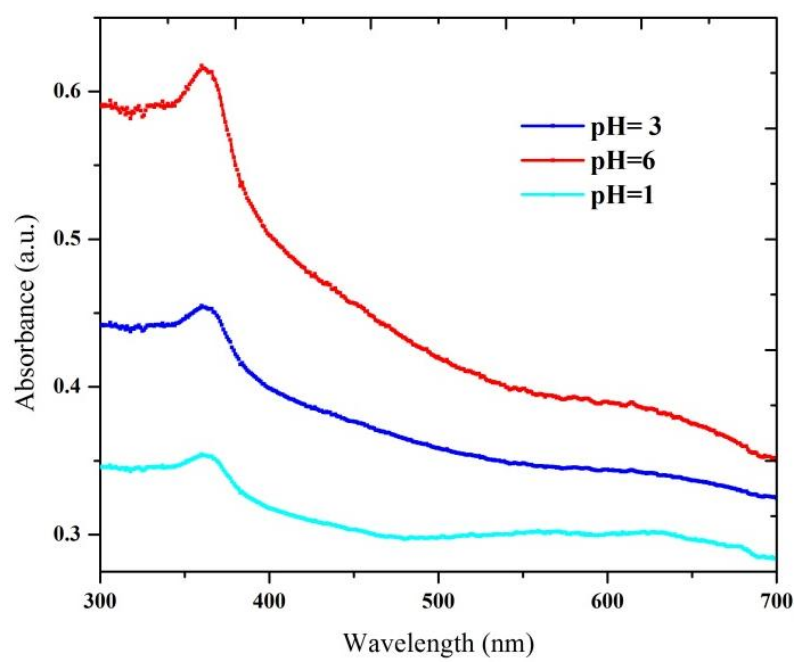

(c)

Figure6: (a) Time-dependent measurements of $\mathrm{ZnO}$ QDs and $\mathrm{ZnO}-$ Graphene dissolution based on Normalized $\mathrm{ZnO}$ content at $\mathrm{pH}=1$, (b) UV-visible absorbance of $\mathrm{ZnO}$ QDs solution after reaching to equilibrium, and (c) UV-visible absorbance of $\mathrm{ZnO}-30 \%$ Graphene solution after reaching to equilibrium (100 mg/L initial mass of ZnO QDs and ZnO Graphene ). 


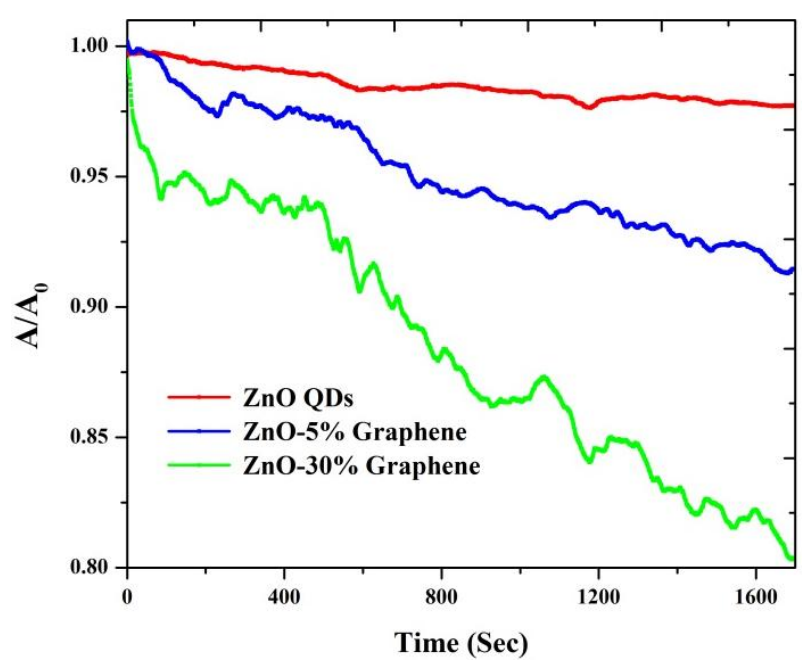

(a)

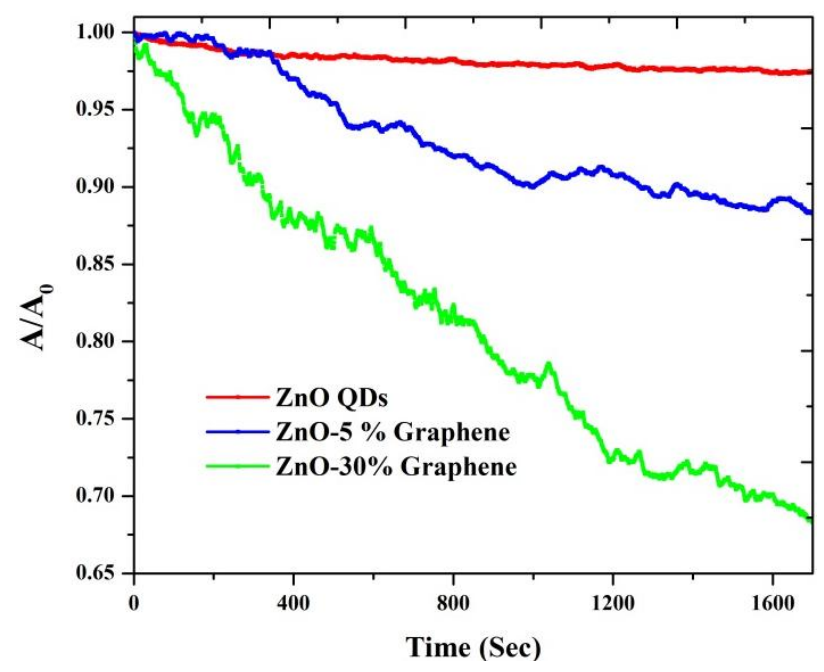

(b)

Figure7: Sedimentation plot of $\mathrm{ZnO}$ QDs, and $\mathrm{ZnO}-$ Graphene hybrid in (a) DMF , and (b) Water. 


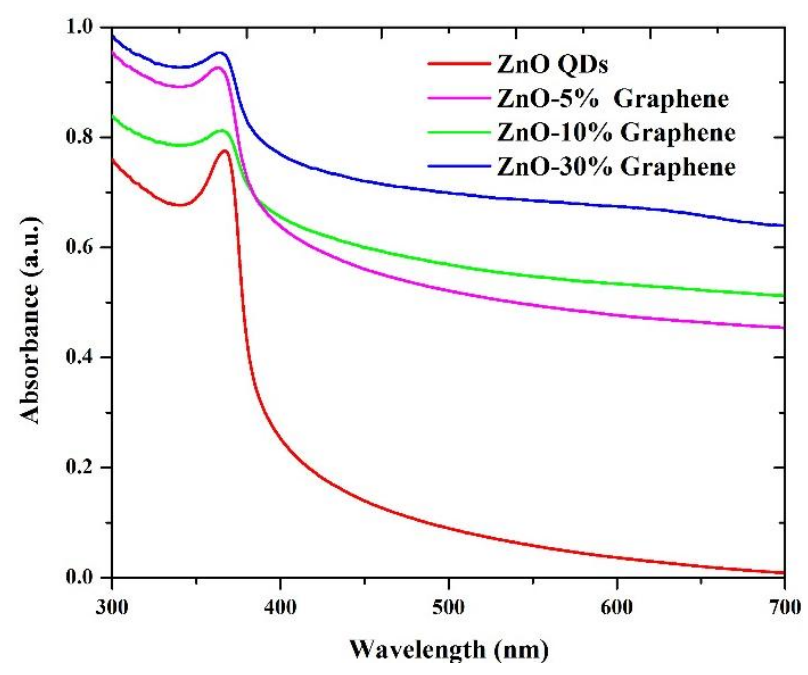

(a)

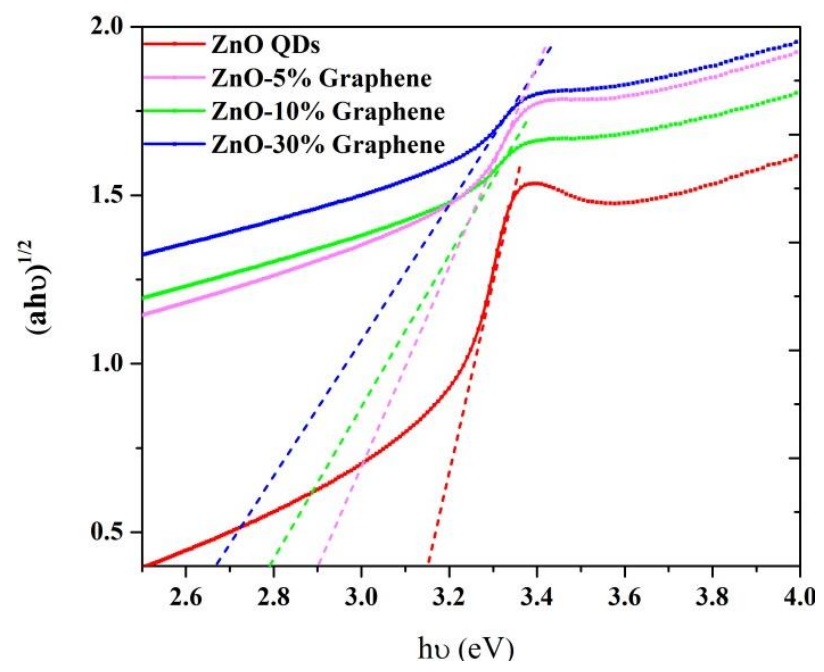

(b)

Figure 8: (a) UV-Visible absorbance of $\mathrm{ZnO}$ QDs and ZnO-Graphene, and (b) The plot of transformed Kubelka-Munk function versus the energy of light. 


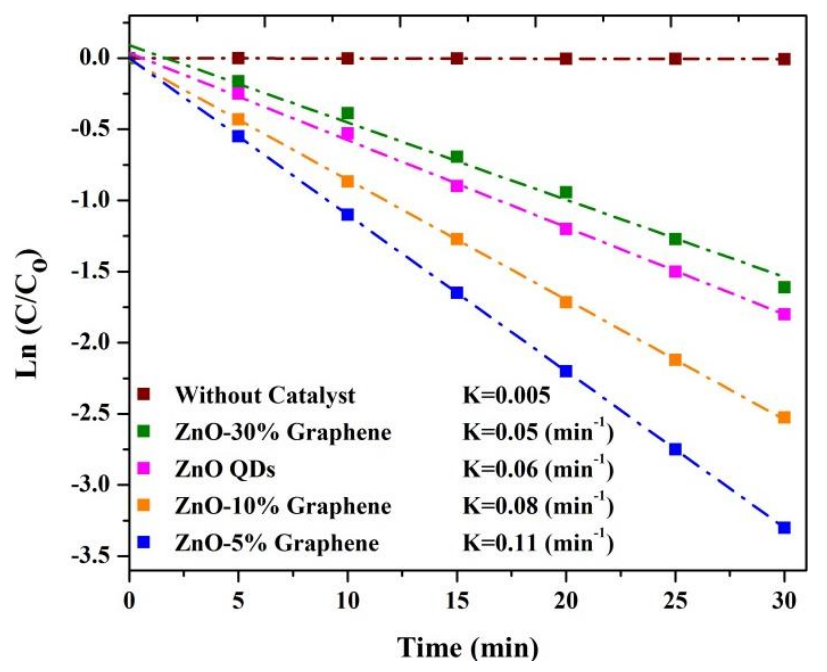

(a)

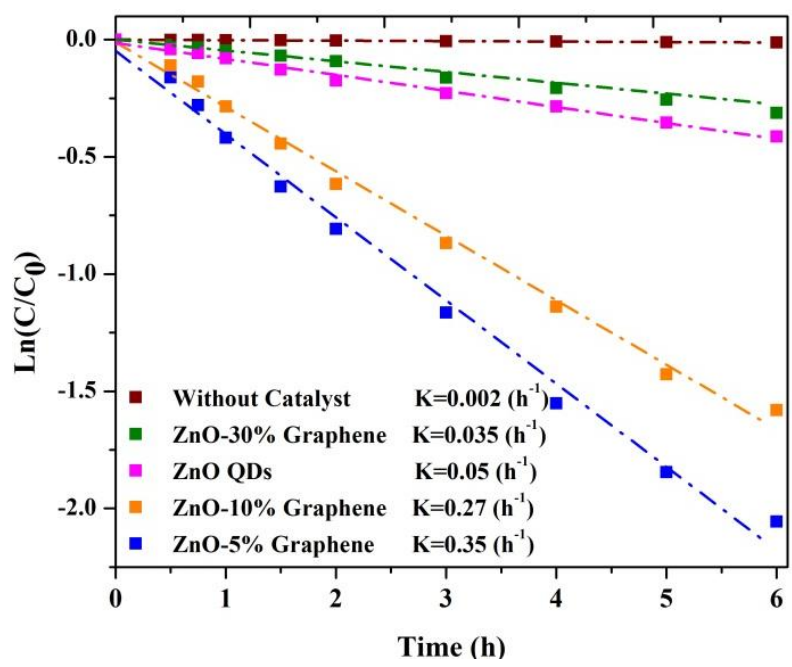

(b)

Figure 9: Kinetic of photocatalytic degradation of MO under (a) UV and (b) Visible light irradiation for $\mathrm{ZnO}$ QDs and $\mathrm{ZnO}-$ Graphene hybrid. 


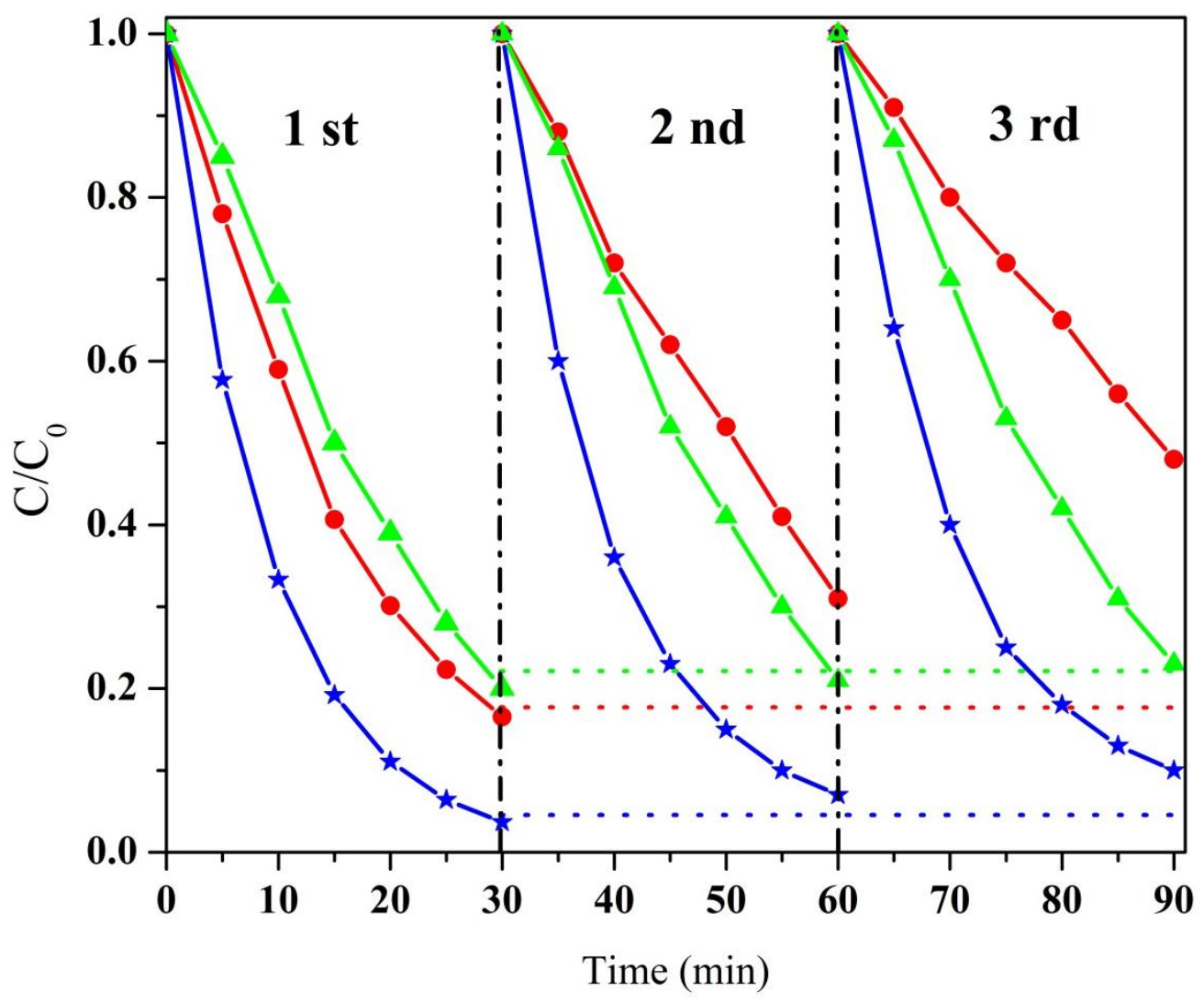

Figure 10: The photostability of $\mathrm{ZnO}$ QDs and $\mathrm{ZnO}-$ Graphene composites by investigation of photocatalytic activity under UV irradiation, $($ Red circle $=\mathrm{ZnO}$ QDs, Green triangle $=\mathrm{ZnO}-30 \%$ Graphene, Blue Star $=$ ZnO-5\% Graphene). 


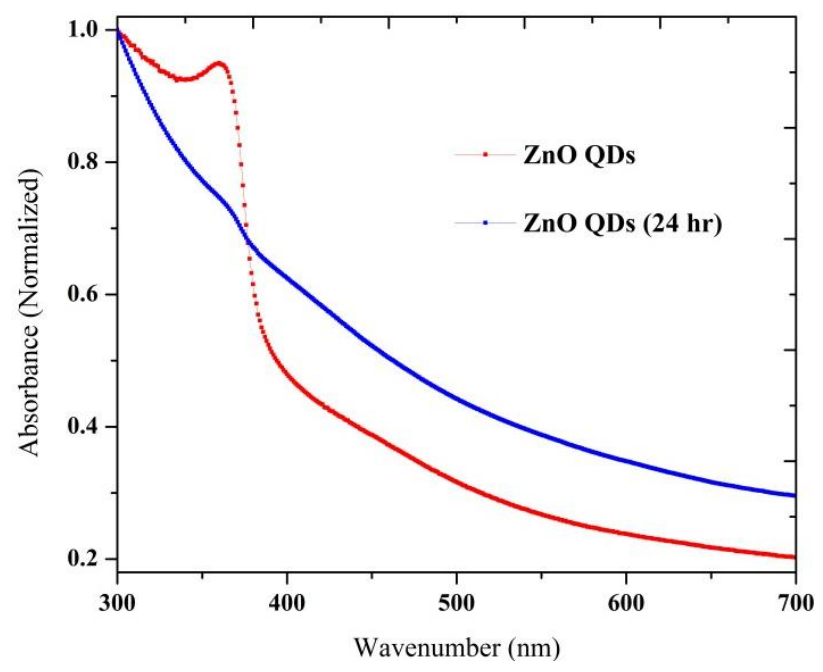

(a)

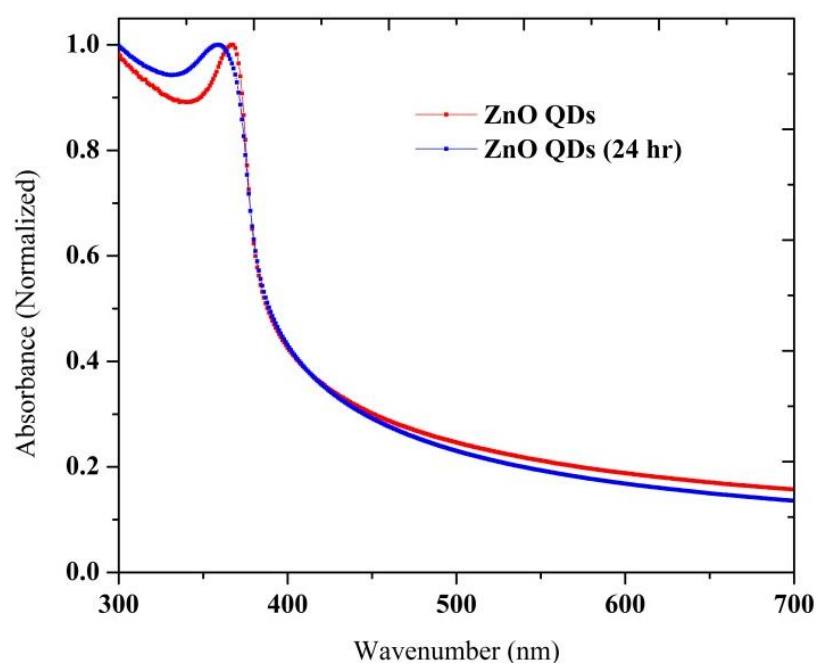

(c)

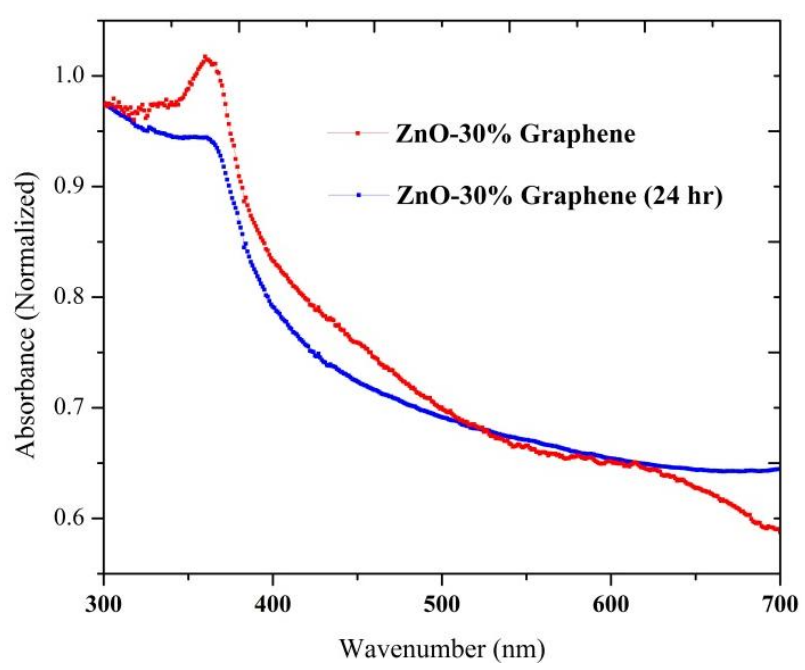

(b)

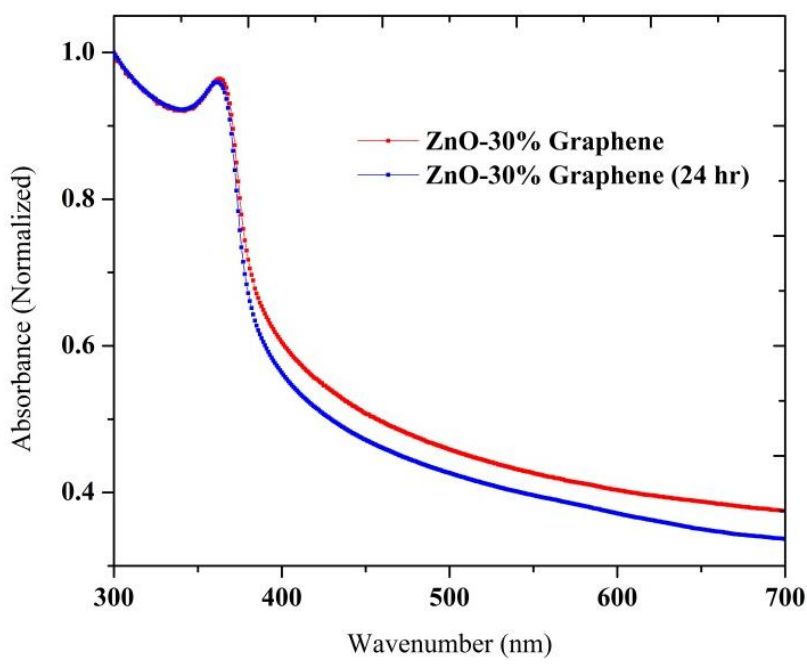

(d)

Figure 11: UV-Visible absorbance of (a) ZnO QDs, (b) ZnO-30\% Graphene in aquous solution,

(c) $\mathrm{ZnO}$ QDs, and (d) ZnO-30\% Graphene in DMF solution before and after 24 UV irradiation. 


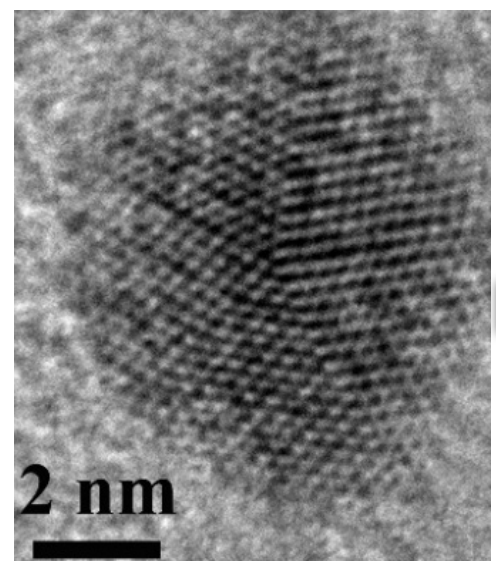

ZnO-Graphene Quantum Dots

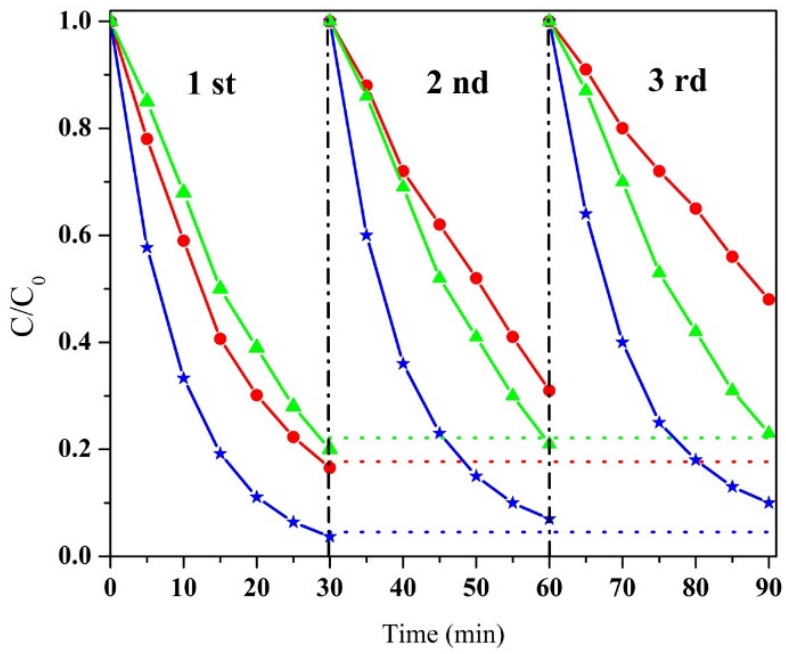

Photocorossion Suppresion of ZnO QDs
Electron-hole Separation- UV light Irradiation

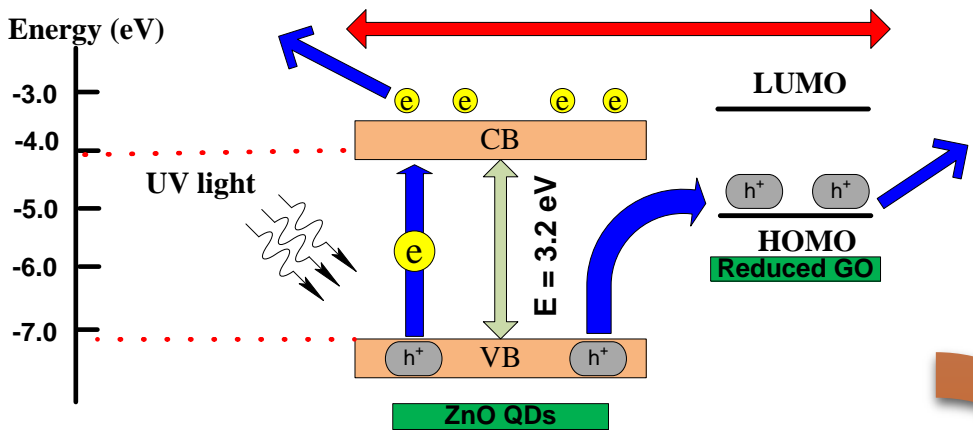

Electron-hole Separation Mechanism

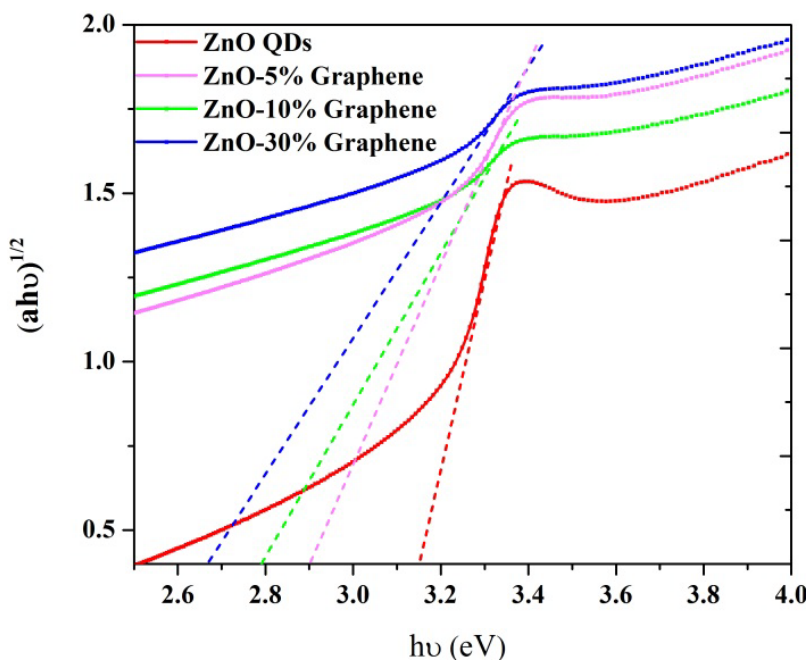

Visible light activation of ZnO-Graphene 